Homology, Homotopy and Applications, vol.8(2), 2006, pp.1-28

\title{
BORDS HOMOTOPIQUES ET MODÈLES DE QUILLEN
}

\author{
THOMAS KAHL, PASCAL LAMBRECHTS AND LUCILE \\ VANDEMBROUCQ
}

(communicated by J. Stasheff)

\begin{abstract}
A homotopy boundary of a finite simply connected CWcomplex $X$ is the boundary of a compact manifold of the homotopy type of $X$. We study the rational homotopy type of the homotopy boundaries of $X$ using Quillen models and we show, in particular, that a large class of homotopy boundaries of $X$ is rationally determined by the rational homotopy type of $X$.

Resumé

Un bord homotopique d'un CW-complexe fini simplement connexe $X$ est, par définition, le bord d'une variété compacte du type d'homotopie de $X$. Nous étudions le type d'homotopie rationnelle des bords homotopiques de $X$ à l'aide des modèles de Quillen et nous montrons, en particulier, qu'une large classe de bords homotopiques de $X$ est rationnellement déterminée par le type d'homotopie rationnelle de $X$.
\end{abstract}

\section{Introduction}

Soit $X$ un CW-complexe fini. On appelle bord homotopique de $X$ de dimension $n$ le bord, $\partial N$, d'une variété compacte $N$ de dimension $n+1$ ayant le type d'homotopie de $X$, autrement dit c'est le bord d'un thickening de $X$. Cette notion apparaît naturellement dans le contexte des plongements: le bord du fibré normal en disques d'une variété $X$ plongée dans une autre est un bord homotopique de $X$. Plus généralement, le bord d'un voisinage régulier d'un polyèdre $X$ plongé à homotopie près dans une variété est un bord homotopique de $X$. En général, le type d'homotopie des bords homotopiques de dimension donnée d'un CW-complexe $X$ ne dépend pas uniquement du type d'homotopie de $X$. Par exemple, le tore $S^{1} \times S^{1}$ et la bouteille de Klein sont deux bords homotopiques de dimension 2 du cercle $S^{1}$. Cependant, sous certaines hypothèses, le type d'homotopie rationnelle d'un bord homotopique ne dépend que du type d'homotopie rationnelle de $X$. Dans [4], le second auteur a montré qu'il en est ainsi de tous les bords homotopiques de $X$ de

P. Lambrechts est Chercheur Qualifié au F.N.R.S.

La recherche de L. Vandembroucq a été partiellement financée par une bourse Lavoisier et par une bourse de la fondation Alexander von Humboldt.

Received November 11, 2004, revised February 12, 2006; published on April 12, 2006.

2000 Mathematics Subject Classification: 55P62.

Key words and phrases: Quillen models, homotopy boundaries, thickenings.

Copyright (C) 2006, International Press. Permission to copy for private use granted. 
dimension supérieure ou égale à $2 \operatorname{dim} X+2$. Notre but est d'étendre ce résultat à une plus large classe de bords homotopiques.

Les bords homotopiques de dimension supérieure ou égale à $2 \operatorname{dim} X+2$ sont, en particulier, des bords homotopiques "suspensions" (c'est-à-dire de la forme $\partial(N \times[0,1])$ où $N$ est un thickening de $X$, cf. la définition dans la section 2) et on pourrait espérer étendre le résultat de [4] à la classe de ces bords. Malheureusement cela n'est pas possible: nous verrons dans la section 8 que $\mathbb{C P}(4)$ admet deux bords homotopiques suspensions qui sont rationnellement différents. Cela nous amène à considérer la classe $\mathcal{S}$ des bords homotopiques "suspensions" de bords homotopiques "à section" (c'est-à-dire dont $X$ est un rétracte, cf. la définition dans la section 2) et nous établissons que tous les bords de cette classe sont rationnellement déterminés par leur dimension et par le type d'homotopie rationnelle de $X$. Notons que la classe $\mathcal{S}$ contient tous les bords homotopiques qui sont des double-suspensions (i.e. de la forme $\partial(N \times[0,1] \times[0,1])$.) De plus, l'exemple mentionné ci-dessus montre que les conditions caractérisantes de la classe $\mathcal{S}$ ne peuvent être affaiblies.

Pour établir ce résultat nous travaillons avec les modèles de Quillen. Rappelons que, si $X$ est un espace simplement connexe, un modèle de Quillen de $X$ est une algèbre de Lie graduée différentielle (LGD) $L=(\mathbb{L}(V), \partial)$ qui détermine le type d'homotopie rationnelle de $X$. Nous montrons dans la section 6 comment construire explicitement à partir d'un tel modèle $L$, une LGD notée $\mathcal{B}^{n}(L)$ qui est un modèle de Quillen de tout bord homotopique de classe $\mathcal{S}$ et dimension $n$ de $X$ (théorème 7.1). On en déduit l'invariance homotopique rationnelle de ces bords homotopiques.

Une des motivations de cette étude du type d'homotopie rationnelle des bords homotopiques est son application à des problèmes de plongements. Si $X$ est un espace simplement connexe ayant le type d'homotopie d'un sous-polyèdre $K$ d'une sphère $S^{n+1}$ alors il existe une somme amalgamée homotopique

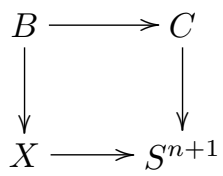

dans laquelle $B$ est un bord homotopique de $X$. Inversement, Browder [2] a démontré que l'existence d'une telle somme amalgamée homotopique vérifiant certaines conditions supplémentaires garantissait l'existence d'un plongement "homotopique" de $X$ dans $S^{n+1}$. La construction du bord homotopique $B$ est souvent une étape préliminaire pour obtenir le diagramme (1) et le modèle de Quillen $\mathcal{B}^{n}(L)$ décrit dans cet article peut être un guide pour cette construction. C'est exactement la démarche qui a été suivie dans [5] pour étudier les plongements homotopiques des 2 -cônes dans une sphère ${ }^{1}$. Notons que si $n>2 \operatorname{dim} X$ alors un argument classique de position générale montre qu'il existe toujours un plongement de $X$ dans $S^{n+1}$. C'est donc l'étude des bords homotopiques hors du rang stable qui est pertinente pour les applications aux plongements et ceci motive la présente extension des résultats

\footnotetext{
${ }^{1}$ Ajouté aux épreuves: Récemment Christophe Boilley a fait d'importants progrès dans ce programme lorsque $\mathrm{X}$ est une variété fermée. Voir sa thèse "Plongements entre variétés lisses à homotopie rationnelle près", UCL, 2005, disponible via http://edoc.bib.ucl.ac.be/ .
} 
de [4]. Nous donnons plus de détails sur cette approche dans la remarque 2 de la section 7 du présent article.

Le texte est organisé de la manière suivante: Les sections 2 et 3 contiennent des définitions et rappels sur les bords homotopiques et les modèles de Quillen. Dans la section 4, nous étudions la cohomologie des bords homotopiques "à section". Les sections 5, 6 et 7 sont consacrées à la modélisation des bords homotopiques. Nous commençons par montrer qu'un modèle de Quillen d'un bord homotopique à section admet une forme très particulière que nous appelons modèle quasi-étrusque. Parmi les modèles quasi-étrusques se distinguent les modèles dits étrusques qui seront décrits explicitement et qui permettront, finalement, de caractériser le type d'homotopie rationnelle des bords homotopiques de classe $\mathcal{S}$. Enfin, la section 8 contient un exemple montrant que nos résultats ne s'étendent pas aux bords homotopiques qui ne sont pas de classe $\mathcal{S}$.

Remerciements. Nous remercions Yves Félix et Greg Lupton pour de fructueuses conversations autour des thèmes de cet article. Le second auteur remercie l'UMR8524 au CNRS à l'Université de Lille-I pour son hospitalité.

\section{Bords homotopiques et thickenings}

Les objets d'étude de ce texte sont les bords homotopiques de CW-complexes finis simplement connexes:

Définition 2.1. Soit $X$ un $C W$-complexe fini simplement connexe. Un bord homotopique de dimension $n$ de $X$ est le bord $\partial N$ d'une variété P.L. (linéaire par morceaux) compacte $N$ de dimension $n+1$ ayant le type d'homotopie de $X$ et tel que $\pi_{1}(\partial N)=0$.

La notion de bord homotopique est étroitement liée à celle de thickening introduite par Stallings et puis Wall [11]: un thickening de dimension $n+1$ d'un CW-

complexe simplement connexe $X$ est une équivalence d'homotopie $\phi: X \stackrel{\simeq}{\rightrightarrows} N$ où $N$ est une variété compacte P.L. de dimension $n+1$ à bord simplement connexe. Un bord homotopique n'est donc rien d'autre que le bord d'un thickening. Deux thickenings $\phi: X \stackrel{\simeq}{\rightrightarrows} N$ et $\phi^{\prime}: X \stackrel{\simeq}{\rightrightarrows} N^{\prime}$ sont dits équivalents s'il existe un PL-homéomorphisme $h: N \stackrel{\cong}{\rightrightarrows} N^{\prime}$ tel que $h \circ \phi$ est homotope à $\phi^{\prime}$. Toujours suivant Wall [11] nous noterons $\mathcal{T}^{n+1}(X)$ l'ensemble des classes d'équivalence de thickenings de $X$ de dimension $n+1$.

On distingue les thickenings remarquables suivants (qui, mis à part les thickenings euclidiens, superstables et ceux de classe $\mathcal{S}$, sont définis dans [11]):

Thickening euclidien. Un thickening euclidien est un thickening obtenu comme voisinage régulier d'un plongement P.L. d'un polyèdre fini du type d'homotopie de $X$ dans un espace euclidien $\mathbb{R}^{n+1}$ (voir [8, p. 33] pour la définition et les propriétés des voisinages réguliers). En vertu de l'unicité du voisinage régulier, ce thickening ne dépend que du choix du polyèdre et de la classe d'isotopie de son plongement. 
Suspension d'un thickening. Si $N$ est un thickening de $X$ alors $N^{\prime}=N \times[0,1]$ est aussi un thickening de $X$ qu'on appelle la suspension du thickening $N$. Cela définit une application $S: \mathcal{T}^{n}(X) \rightarrow \mathcal{T}^{n+1}(X)$. Par construction, on a

$$
\partial N^{\prime}=(\partial N \times[0,1]) \cup_{\partial N \times\{0,1\}}(N \times\{0,1\}) .
$$

Autrement dit $\partial N^{\prime}$ est le "double mapping cylindre" de deux copies de l'inclusion $\partial N \hookrightarrow N$. Donc $\partial N^{\prime}$ est le double de la variété $N$.

Thickening à section. On dit qu'un thickening $\phi: X \stackrel{\cong}{\rightarrow} N$ admet une section s'il existe une application $\nu: X \rightarrow \partial N$ telle que la composée $X \stackrel{\nu}{\rightarrow} \partial N \hookrightarrow N$ est homotope à $\phi$.

Thickening stable et superstable. Dans [11, Section 5], un thickening $N$ de $X$ est dit stable si $\operatorname{dim} N \geqslant 2 \operatorname{dim} X+1$. Nous dirons que $N$ est un thickening superstable $\sin \operatorname{dim} N \geqslant 2 \operatorname{dim} X+2$.

Thickening trivial Un thickening trivial est un thickening euclidien et stable. Tout CW-complexe $X$ de dimension $m$ admet un unique thickening trivial en chaque dimension $n+1 \geqslant 2 m+1$ (voir [11, Section 3]). Notons que, contrairement à ce que la terminologie suggère, lorsque $X$ est une variété fermée, la variété à bord $X \times D^{k}$ n'est pas nécessairement un thickening trivial de $X$. Ainsi $\mathbb{C} P(2) \times D^{k}$ n'est pas un thickening trivial de $\mathbb{C} P(2)$ car le fibré normal du plongement de $\mathbb{C} P(2)$ dans un espace euclidien n'est pas trivial.

Outre ces notions classiques de thickenings, nous considérerons tout au long de ce travail la classe particulière suivante:

Thickening de classe $\mathcal{S}$. Un thickening $N$ de $X$ est dit de classe $\mathcal{S}$ s'il est la suspension d'un thickening à section de $X$. Nous montrons ci-dessous que les doubles suspensions de thickenings ainsi que les thickenings superstables sont de classe $\mathcal{S}$.

Nous appellerons bord homotopique euclidien (resp. trivial, suspension, à section, stable, superstable ou de classe $\mathcal{S}$ ) le bord d'un thickening euclidien (resp. trivial, suspension, à section, stable, superstable ou de classe $\mathcal{S}$ ).

Voici quelques résultats classiques qui relient ces différentes classes de thickenings. Nous supposons toujours dans la suite de ce travail que la dimension $n+1 \mathrm{du}$ thickening est supérieure à 6 et à $\operatorname{dim} X+2$. Parmi les énoncés suivants, ceux qui ne sont pas immédiats sont démontrés dans [11].

- La suspension d'un thickening $\phi: X \stackrel{\simeq}{\rightarrow} N$ est un thickening à section, une section étant donnée par la composée $X \stackrel{\simeq}{\rightrightarrows} N=N \times\{0\} \hookrightarrow \partial(N \times[0,1])$. Donc la double suspension d'un thickening est de classe $\mathcal{S}$.

- Un thickening superstable est aussi un thickening de classe $\mathcal{S}$. En effet Wall ([11, Section 5]) montre que la suspension $S: \mathcal{T}^{n}(X) \rightarrow \mathcal{T}^{n+1}(X)$ est bijective pour $n \geqslant 2 \operatorname{dim} X+1$ et surjective pour $n \geqslant 2 \operatorname{dim} X$. En conséquence, un thickening superstable est dans l'image de $S \circ S$ et, par suite, est la suspension d'un thickening à section. 
Exemples:

(1) Un exemple de thickening sans section est le suivant (appelé thickening de Hopf de $S^{2}$ ): on considère le fibré de Hopf $\eta: \mathbb{C} \rightarrow E \rightarrow S^{2}$ en droites complexes et $D^{2} \rightarrow N \rightarrow S^{2}$ le fibré en disque associé. La variété à bord $N$ est un thickening de $S^{2}$ et son bord est $\partial N=S^{3}$. Il est immédiat que ce thickening n'admet pas de section.

(2) Dans le rang stable, $S^{2}$ admet les deux thickenings suivants: le thickening $N_{0}=S^{2} \times D^{k+1}$, qui est trivial car il correspond au plongement usuel $S^{2} \subset \mathbb{R}^{k+3}$, et le thickening $N^{\prime}$ associé au fibré orthogonal en disques non trivial sur $S^{2}$ (obtenu par suspension du fibré de Hopf $\eta: \mathbb{C} \rightarrow E \rightarrow S^{2}$ ). Le bord du thickening $N_{0}$ est $\partial N_{0}=S^{2} \times S^{k}$ tandis que le bord de $N^{\prime}$ est le fibré orthogonal non trivial en sphères $S^{k}$ sur $S^{2}$. Notons, par exemple, que pour $k=1, \partial N^{\prime}=S^{3}$.

\section{Notations et rappels sur les modèles de Quillen}

Dans cette section, nous fixons nos notations et nous faisons quelques rappels sur les modèles de Quillen. Pour la théorie complète nous renvoyons le lecteur à [10], [1] ou [3]. Soit $V$ un espace vectoriel gradué sur un corps k. Par convention, $V^{k}=V_{-k}$. La $n$-ième suspension de $V$ est l'espace vectoriel gradué $s^{n} V$ défini par

$$
\left(s^{n} V\right)^{k}=V^{k+n} \text {. }
$$

Si $\left\{a_{1}, \ldots, a_{r}\right\}$ est une famille d'éléments homogènes de $V$ alors $\left\langle a_{1}, \ldots, a_{r}\right\rangle$ désigne le sous-espace vectoriel engendré par cette famille. Le dual de $V$ est $\# V:=\operatorname{hom}(V, \mathbf{k})$ muni de la graduation $(\# V)^{k}=\operatorname{hom}\left(V^{-k}, \mathbf{k}\right)$. La dualité entre $V$ et \#V est notée

$$
\langle-,-\rangle: V \otimes \# V \rightarrow \mathbf{k}, v \otimes f \mapsto\langle v, f\rangle=f(v) .
$$

Dans ce travail nous considérerons des algèbres de Lie graduées différentielles (LGD) connexes sur le corps $\mathbb{Q}([\mathbf{1 0}, 0.4])$. Un modèle de Quillen $(L, \partial)$ est une LGD telle que $L$ est une algèbre de Lie graduée libre $\mathbb{L}(V)$ sur un espace vectoriel gradué $V$. La donnée de $V \subset L$ telle que $L=\mathbb{L}(V)$ est appelé un choix des indécomposables de $L$. L'espace des indécomposables d'une LGD $(L, \partial)$ est $Q L:=L /[L, L]$ et est muni d'une différentielle induite $Q \partial$. Si $W \subset L$ est un sous-espace vectoriel de l'algèbre de Lie graduée $L$ nous désignons par $(W)$ l'idéal engendré par $W$. L'algèbre de Lie graduée libre $\mathbb{L}(V)$ est filtrée par la longueur des crochets, $\{\mathbb{L} \geqslant k(V)\}$. De même l'algèbre tensorielle $T(V)$ est filtrée par la longueur des mots et on pose $T^{+}(V)=$ $T \geqslant 1(V)$.

Dans [7], Quillen associe à tout espace simplement connexe $X$ une LGD $\lambda(X)$ dont la classe de quasi-isomorphisme détermine le type d'homotopie rationnelle de $X$. Un modèle de Quillen de $X$ est la donnée d'un modèle de Quillen $(L, \partial)$ quasiisomorphe à $\lambda(X)$. D'après le résultat principal de $[6]$ cette définition équivaut à celle de [10, III.3]. Un modèle de Quillen $(L, \partial)$ est dit minimal si $\partial(L) \subset[L, L]$. On peut également définir le modèle de Quillen d'une application continue entre espaces simplement connexes. On a un isomorphisme $s H(Q L, \partial Q) \cong \tilde{H}_{*}(X ; \mathbb{Q})([\mathbf{3}, 24(\mathrm{~b})])$ et lorsque le modèle est minimal la partie quadratique $\partial_{2}$ de la différentielle $\partial$ est reliée au cup produit en cohomologie (voir [10, III.3(9)]). La dimension formelle de $L$ est l'entier $\sup \left\{k:(s H(Q L, \partial Q))_{k} \neq 0\right\}$. Donc la dimension formelle d'un modèle 
de Quillen d'un espace $X$ est exactement la dimension cohomologique rationnelle de $X$.

\section{Cohomologie des bords homotopiques}

Dans cette section $\mathbf{k}$ désigne un corps commutatif. Si $\phi: X \stackrel{\simeq}{\rightarrow} N$ est un thickening d'inverse homotopique $\psi: N \stackrel{\simeq}{\rightrightarrows} X$ alors $H^{*}(\partial N ; \mathbf{k})$ admet une structure de $H^{*}(X ; \mathbf{k})-$ module induite par l'application composée $\partial N \hookrightarrow N \stackrel{\psi}{\rightarrow} X$. On a d'autre part une structure de $H^{*}(X ; \mathbf{k})$-module évidente sur $\# s^{n} H^{*}(X ; \mathbf{k})$.

Théorème 4.1. Soit $X$ un $C W$-complexe fini simplement connexe.

(A) Si $\partial N$ est un bord homotopique à section de dimension $n$ de $X$ alors il existe un isomorphisme de $H^{*}(X ; \mathbf{k})$-modules

$$
H^{*}(\partial N ; \mathbf{k}) \cong H^{*}(X ; \mathbf{k}) \oplus \# s^{n} H^{*}(X ; \mathbf{k})
$$

(B) Si $\partial N$ est un bord homotopique de classe $\mathcal{S}$ de dimension $n$ de $X$ alors (2) est un isomorphisme d'algèbre où la structure d'algèbre du membre de droite est caractérisée par sa structure de $H^{*}(X ; \mathbf{k})$-module et par l'équation

$$
\# s^{n} H^{*}(X ; \mathbf{k}) \cdot \# s^{n} H^{*}(X ; \mathbf{k})=0 .
$$

Preuve. (A) Dans cette preuve nous écrirons $H^{*}(-)$ pour $H^{*}(-; \mathbf{k})$. Notons $\nu: X \rightarrow$ $\partial N$ la section et $j$ l'inclusion $\partial N \hookrightarrow N$. Puisque $j \circ \nu$ est une équivalence d'homotopie, la longue suite exacte de cohomologie de la paire $(N, \partial N)$ donne une courte suite exacte scindée:

$$
0 \longrightarrow H^{*}(N) \stackrel{H^{*}(j)}{\longrightarrow} H^{*}(\partial N) \stackrel{\delta}{\longrightarrow} H^{*+1}(N, \partial N) \longrightarrow 0 .
$$

L'équivalence d'homotopie $\phi: X \stackrel{\cong}{\rightrightarrows} N$ induit un isomorphisme $\phi^{*}: H^{*}(N) \stackrel{\cong}{\rightarrow}$ $H^{*}(X)$. Comme $X$ est simplement connexe, il en est de même de $N$. Par conséquent $N$ est une variété orientable et par dualité de Poincaré on a un isomorphisme

$$
H^{k+1}(N, \partial N) \stackrel{-\cap[N]}{\cong} H_{n-k}(N) .
$$

Cet isomorphisme, tout comme les isomorphismes

$$
H_{n-k}(N) \cong\left(\# H^{*}(N)\right)^{-n+k} \cong\left(s^{-n} \# H^{*}(N)\right)^{k} \cong\left(\# s^{n} H^{*}(X)\right)^{k},
$$

respecte les stuctures de $H^{*}(X)$-modules. On a ainsi une courte suite exacte e $H^{*}(X)$-modules

$$
0 \longrightarrow H^{*}(X) \longrightarrow H^{*}(\partial N) \longrightarrow \# s^{n} H^{*}(X) \longrightarrow 0
$$

scindée par $H^{*}(\nu)$. Ceci démontre le point $(A)$.

(B) Par hypothèse $N$ est la suspension d'un thickening $\phi^{\prime}: X \stackrel{\simeq}{\rightarrow} N^{\prime}$ à section $\nu^{\prime}: X \rightarrow \partial N^{\prime}$. Notons $j^{\prime}: \partial N^{\prime} \hookrightarrow N^{\prime}$ l'inclusion et considérons le cube commutatif suivant dans lequel les faces supérieure et inférieure sont des sommes amalgamées et 
l'application $\tau: X \rightarrow N^{\prime} \cup_{\partial N^{\prime}} N^{\prime}$ est l'application induite entre ces sommes amalgamées:

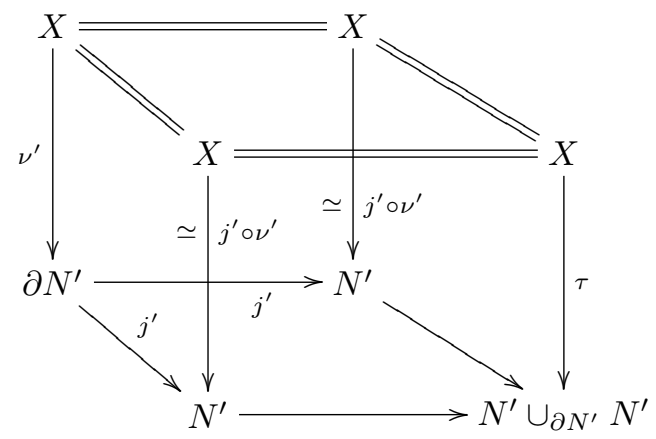

Soit $h: N^{\prime} \cup_{\partial N^{\prime}} N^{\prime} \rightarrow \partial N$ un inverse de l'équivalence d'homotopie

$$
\partial N=\left(\partial N^{\prime} \times[0,1]\right) \cup_{\partial N^{\prime} \times\{0,1\}}\left(N^{\prime} \times\{0,1\}\right) \stackrel{\sim}{\rightarrow} N^{\prime} \cup_{\partial N^{\prime}} N^{\prime},
$$

induite par la projection $\partial N^{\prime} \times[0,1] \rightarrow \partial N^{\prime}$. On vérifie que $\nu:=h \circ \tau: X \rightarrow \partial N$ est une section du thickening $N$. En prenant les cofibres homotopiques des applications verticales du cube ci-dessus (nous noterons $C_{f}$ la cofibre homotopique d'une application $f$ ) on obtient la somme amalgamée suivante

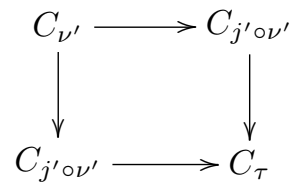

Comme $C_{j^{\prime} \circ \nu^{\prime}}$ est contractile, $C_{\tau}$, et par suite $C_{\nu}$, a le type d'homotopie d'une suspension. Cela entraîne que tous les produits de $\tilde{H}^{*}\left(C_{\nu}\right)$ sont nuls.

Démontrons que (2) est un isomorphisme d'algèbre. Comme nous savons par le point $(A)$ que c'est un isomorphisme de $H^{*}(X)$-module, il suffit de vérifier que $K \cdot K=0$ où $K \subset H^{*}(\partial N)$ est l'image de $\# s^{n} H^{*}(X)$ par l'isomorphisme (2). Notons $i: \partial N \hookrightarrow C_{\nu}=\partial N \cup_{\nu} C X$ l'inclusion dans le mapping cone. Comme $K=$ $\operatorname{ker}\left(H^{*}(\nu)\right)$, nous trouvons que $K$ est aussi l'image de $\tilde{H}^{*}\left(C_{\nu}\right)$ par $H^{*}(i)$. Ainsi $K \cdot K=0$ résulte de $\tilde{H}^{*}\left(C_{\nu}\right) \cdot \tilde{H}^{*}\left(C_{\nu}\right)=0$.

\section{Modèle de Quillen d'un bord homotopique à section}

Dans la perspective de caractériser les modèles de Quillen des bords homotopiques d'un CW-complexe fini simplement connexe nous introduisons les notions suivantes de modèles étrusques et quasi-étrusques:

Définition 5.1. Un modèle de Quillen $L$ est dit quasi-étrusque s'il existe des espaces vectoriels gradués de dimension finie $V$ et $V^{*}$ et un élément $\mu$ tels que $L \cong\left(\mathbb{L}\left(V \oplus V^{*} \oplus \mathbb{Q} \cdot \mu\right), \partial\right)$ avec

(i) $\partial(V) \subset \mathbb{L}(V)$; 
(ii) $\partial\left(V^{*}\right) \subset I$ où $I:=\left(V^{*}\right)$ est l'idéal engendré par $V^{*}$;

(iii) $\partial(\mu)=\sum_{i=1}^{l}\left[v_{i}, v_{i}^{*}\right]+\xi$ où $\left\{v_{i}\right\}_{1 \leqslant i \leqslant l}$ et $\left\{v_{i}^{*}\right\}_{1 \leqslant i \leqslant l}$ sont des bases homogènes respectivement de $V$ et de $V^{*}$ et $\xi \in[I, I]$.

Si de plus $\xi=0$, c'est-à-dire si $\partial(\mu)=\sum_{i}\left[v_{i}, v_{i}^{*}\right]$, on dit alors que le modèle $L$ est étrusque.

Le modèle $(\mathbb{L}(V), \partial)$ est appelé un socle du modèle (quasi-)étrusque $L$.

La famille $\left\{v_{i}, v_{i}^{*}, \mu\right\}_{1 \leqslant i \leqslant l}$ est appelée une base adaptée de ce modèle (quasi-) étrusque.

\section{Remarques.}

1. Puisque $\partial\left(V^{*} \oplus \mathbb{Q} . \mu\right) \subset I$, un modèle quasi-étrusque se rétracte sur son socle. Plus précisément on a des morphismes de LGD

$$
(\mathbb{L}(V), \partial) \stackrel{\sigma}{\longrightarrow}\left(\mathbb{L}\left(V \oplus V^{*} \oplus \mathbb{Q} \cdot \mu\right), \partial\right) \stackrel{\text { pr }}{\longrightarrow}(\mathbb{L}(V), \partial),
$$

où $\sigma$ est une extension libre et pr la projection évidente.

2. Il est clair que $\mu$ représente l'élément de plus haut degré dans l'homologie des indécomposables $H_{*}(Q L, Q \partial)$, et donc, si $\operatorname{deg}(\mu)=n-1$, alors $L$ est de dimension formelle $n$.

3. Si un modèle quasi-étrusque minimal $L$ est le modèle d'un espace $X$ alors on peut démonter que $H^{*}(X ; \mathbb{Q})$ est à dualité de Poincaré. Ceci provient essentiellement de la formule (iii) pour $\partial(\mu)$ et de l'interprétation de la partie quadratique de la différentielle du modèle de Quillen en termes de cup-produit dans la cohomologie de l'espace. L'élément $\mu$ correspond alors à la classe fondamentale de $H^{*}(X ; \mathbb{Q})$.

4. Le socle d'un modèle quasi-étrusque n'est en général pas unique. Par exemple

$$
\left(\mathbb{L}\left(x_{1}, y_{3}, z_{5}, t_{7}, \mu_{9}\right), \partial(x)=\partial(y)=\partial(z)=\partial(t)=0, \partial(\mu)=[x, t]+[y, z]\right),
$$

est un modèle étrusque dont $\left(\mathbb{L}\left(x_{1}, y_{3}\right), 0\right)$ et $\left(\mathbb{L}\left(x_{1}, z_{5}\right), 0\right)$ sont deux socles non isomorphes (les indices des générateurs désignent leurs degrés).

5. À la fin de la section 6 nous donnons un exemple d'un modèle quasi-étrusque minimal qui n'est pas isomorphe à un modèle étrusque. Notons cependant que par un résultat classique de J. Stasheff [9], tout modèle quasi-étrusque minimal est isomorphe à un modèle de Quillen minimal satisfaisant la condition (iii) de la définition d'un modèle étrusque.

Les définitions ci-dessus sont motivées par le résultat suivant:

Théorème 5.2. Soit $X$ un $C W$-complexe fini simplement connexe. Tout bord homotopique à section de $X$ admet un modèle de Quillen minimal quasi-étrusque dont un socle est un modèle minimal de $X$. Plus précisément le diagramme

$$
X \stackrel{\nu}{\longrightarrow} \partial N \stackrel{j}{\longrightarrow} N \text {, }
$$

où $\nu$ est une section du bord homotopique admet comme modèle de Quillen un 
diagramme

$$
(\mathbb{L}(V), \partial) \stackrel{\sigma}{\longrightarrow}\left(\mathbb{L}\left(V \oplus V^{*} \oplus \mathbb{Q} \cdot \mu\right), \partial\right) \stackrel{\operatorname{pr}}{\longrightarrow}(\mathbb{L}(V), \partial),
$$

où $\sigma$ est une extension libre, pr est la projection évidente, $(\mathbb{L}(V), \partial)$ est un modèle minimal de $X$ et le terme du milieu est un modèle quasi-étrusque minimal.

Avant de démontrer ce théorème, nous établissons le résultat préliminaire suivant qui sera également utile par la suite.

Proposition 5.3. Soient $X$ et $Y$ deux espaces 1 -connexes et $f: X \rightarrow Y$ et $g: Y \rightarrow$ $Z$ deux applications telles que $g \circ f$ est une équivalence d'homotopie. Le diagramme

$$
X \stackrel{f}{\rightarrow} Y \stackrel{g}{\rightarrow} Z
$$

admet un modèle de Quillen de la forme

$$
(\mathbb{L}(V), \partial) \stackrel{\sigma}{\longmapsto}(\mathbb{L}(V \oplus W), \partial) \stackrel{\operatorname{pr}}{\longrightarrow}(\mathbb{L}(V), \partial),
$$

où $\sigma$ est une extension libre, pr est la projection évidente, $(\mathbb{L}(V), \partial)$ est un modèle minimal de $X$ et $(\mathbb{L}(V \oplus W), \partial)$ est un modèle minimal de $Y$.

Preuve. Soit $L \stackrel{\varphi}{\rightarrow} L^{\prime} \stackrel{\psi}{\rightarrow} L^{\prime \prime}$ un modèle d'algèbre de Lie différentielle de

$$
X \stackrel{f}{\rightarrow} Y \stackrel{g}{\rightarrow} Z
$$

et soit $\mu:(\mathbb{L}(V), \partial) \stackrel{\sim}{\rightarrow} L$ un modèle libre minimal. Factorisons $\varphi \circ \mu$ en une extension libre minimale $\alpha:(\mathbb{L}(V), \partial) \longmapsto(\mathbb{L}(V \oplus W), D)$ et une équivalence faible $\mu^{\prime}:(\mathbb{L}(V \oplus W), D) \stackrel{\sim}{\rightarrow} L^{\prime}$. Considérons le diagramme commutatif

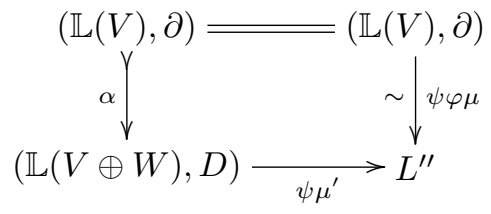

Par le lemme de relèvement, il existe un morphisme de LGD

$$
p:(\mathbb{L}(V \oplus W), D) \rightarrow(\mathbb{L}(V), \partial),
$$

tel que $p \circ \alpha=i d$ et $\psi \varphi \mu p \sim \psi \mu^{\prime}($ rel. $\mathbb{L}(V))$. Cela implique que

$$
(\mathbb{L}(V), \partial) \stackrel{\alpha}{\longmapsto}(\mathbb{L}(V \oplus W), D) \stackrel{p}{\longrightarrow}(\mathbb{L}(V), \partial),
$$

est un modèle de $X \stackrel{f}{\rightarrow} Y \stackrel{g}{\rightarrow} Z$. Considérons l'isomorphisme d'algèbre de Lie

$$
\beta: \mathbb{L}(V \oplus W) \rightarrow \mathbb{L}(V \oplus W),
$$

défini $\operatorname{par} \beta(v)=v$ et $\beta(w)=w+p(w)$. Posons $\partial:=\beta^{-1} D \beta$ et $\sigma:=\beta \alpha$. De cette façon, $\partial$ est une différentielle d'algèbre de Lie sur $\mathbb{L}(V \oplus W), \beta$ est un isomorphisme de LGD et $\sigma$ est une extension libre. Cela montre que

$$
(\mathbb{L}(V), \partial) \stackrel{\sigma}{\longmapsto}(\mathbb{L}(V \oplus W), \partial) \stackrel{\operatorname{pr}}{\longrightarrow}(\mathbb{L}(V), \partial),
$$

où pr est la projection évidente est un modèle de $X \stackrel{f}{\rightarrow} Y \stackrel{g}{\rightarrow} Z$. Comme pr commute 
à la différentielle, $\partial(W)$ est inclus dans ker pr c'est-à-dire dans l'idéal de $\mathbb{L}(V \oplus W)$ engendré par $W$. L'extension $\alpha$, et par suite $\sigma$, étant minimale, il s'ensuit que la différentielle sur $W$ est décomposable. La minimalité de $(\mathbb{L}(V), \partial)$ assure finalement la minimalité de $(\mathbb{L}(V \oplus W), \partial)$.

\section{Preuve du théorème 5.2.}

Soit $\partial N$ un bord homotopique de $X$ à section $\nu$ et de dimension $n$. D'après la proposition précédente, le diagramme

$$
X \stackrel{\nu}{\longrightarrow} \partial N \stackrel{j}{\longrightarrow} N \text {, }
$$

admet un modèle de Quillen de la forme

$$
(\mathbb{L}(V), \partial) \stackrel{\sigma}{\longmapsto}(\mathbb{L}(V \oplus W), \partial) \stackrel{\operatorname{pr}}{\longrightarrow}(\mathbb{L}(V), \partial),
$$

où $(\mathbb{L}(V), \partial)$ et $L:=(\mathbb{L}(V \oplus W), \partial)$ sont des modèles de Quillen minimaux de $X$ et de $\partial N$ respectivement, $\sigma$ est une extension libre et pr est la projection évidente.

L'interprétation des indécomposables du modèle de Quillen minimal ([3, 24(b)]) donne un isomorphisme:

$$
\psi: V \oplus W \stackrel{\cong}{\longrightarrow} \tilde{H}_{*}(\partial N ; \mathbb{Q}),
$$

de degré +1 tel que $\psi(V)=H_{*}(\nu)\left(\tilde{H}_{*}(X ; \mathbb{Q})\right)$ et $\psi(W)=\operatorname{ker} H_{*}(j)$. Soit $\mu \in$ $W_{n-1}$ tel que $\psi(\mu)$ est une classe fondamentale de la variété $\partial N$ et soit $V^{*}$ un supplémentaire homogène de $\mathbb{Q} \cdot \mu$ dans $W$. Ainsi $L=\left(\mathbb{L}\left(V \oplus V^{*} \oplus \mathbb{Q} \cdot \mu\right), \partial\right)$. On a $\partial(V) \subset \mathbb{L}(V)$ et, comme la projection pr commute à la différentielle, $\partial\left(V^{*} \oplus \mathbb{Q} . \mu\right)$ est inclus dans ker pr, c'est-à-dire dans l'idéal de $L$ engendré par $V^{*} \oplus \mathbb{Q} . \mu$. Soit $I:=\left(V^{*}\right)$ l'idéal dans $L$ engendré par $V^{*}$. Alors $\partial\left(V^{*} \oplus \mathbb{Q} . \mu\right) \subset I$ pour des raisons de degrés. En particulier $\partial\left(V^{*}\right) \subset I$.

Par le théorème 4.1-A et par dualité de Poincaré il existe des bases $\left\{v_{i}\right\}_{1 \leqslant i \leqslant l}$ de $V$ et $\left\{v_{i}^{*}\right\}_{1 \leqslant i \leqslant l}$ de $V^{*}$ telles que la diagonale réduite

$$
\bar{\Delta}_{*}: \tilde{H}_{*}(\partial N ; \mathbb{Q}) \rightarrow \tilde{H}_{*}(\partial N ; \mathbb{Q}) \otimes \tilde{H}_{*}(\partial N ; \mathbb{Q}),
$$

vérifie

$$
\bar{\Delta}_{*}(\psi(\mu))=\psi\left(\sum_{i=1}^{l}\left(v_{i} \otimes v_{i}^{*}+(-1)^{\left|v_{i}\right|} v_{i}^{*} \otimes v_{i}\right)+\sum_{p, q} \alpha_{p, q} v_{p}^{*} \otimes v_{q}^{*}\right),
$$

avec $\alpha_{p, q} \in \mathbb{Q}$. Le lien entre la diagonale et la partie quadratique $\partial_{2}$ de la différentielle $\partial(\operatorname{voir}[\mathbf{1 0}, \mathrm{III} .3(9)])$ donne

$$
\partial \mu=\sum_{i=1}^{l}\left[v_{i}, v_{i}^{*}\right]+\xi^{\prime}
$$

avec $\xi^{\prime} \in\left[V^{*}, V^{*}\right]+\mathbb{L}^{\geqslant 3}\left(V \oplus V^{*}\right)$. Notons que $\xi^{\prime} \in I$ car $\partial \mu$ et $\sum_{i=1}^{l}\left[v_{i}, v_{i}^{*}\right]$ appartiennent à $I$. Autrement dit, $\xi^{\prime}=\xi_{3}+\epsilon_{3}$ avec $\xi_{3} \in[I, I]$ et $\epsilon_{3} \in I \cap \mathbb{L} \geqslant 3\left(V \oplus V^{*}\right)$.

Nous allons démontrer par récurrence sur $r \geqslant 3$ qu'il existe une différentielle $\partial_{r}$ sur $\mathbb{L}\left(V \oplus V^{*} \oplus \mathbb{Q} . \mu\right)$ telle que 
1. $\partial_{r}(V) \subset \mathbb{L}(V)$

2. $\partial_{r}\left(V^{*} \oplus \mathbb{Q} \cdot \mu\right) \subset I=\left(V^{*}\right)$

3. $\partial_{r}(\mu)=\sum_{i=1}^{l}\left[v_{i}, v_{i}^{*}\right]+\xi_{r}+\epsilon_{r}$ avec $\xi_{r} \in[I, I]$ et $\epsilon_{r} \in I \cap \mathbb{L}^{\geqslant r}\left(V \oplus V^{*}\right)$

ainsi que des isomorphismes $\lambda_{r}:(\mathbb{L}(V), \partial) \rightarrow\left(\mathbb{L}(V), \partial_{r}\right)$ et

$$
\Lambda_{r}:\left(\mathbb{L}\left(V \oplus^{*} \oplus \mathbb{Q} \cdot \mu\right), \partial\right) \rightarrow\left(\mathbb{L}\left(V \oplus^{*} \oplus \mathbb{Q} \cdot \mu\right), \partial_{r}\right),
$$

tels que le diagramme suivant d'algèbres de Lie différentielles commute

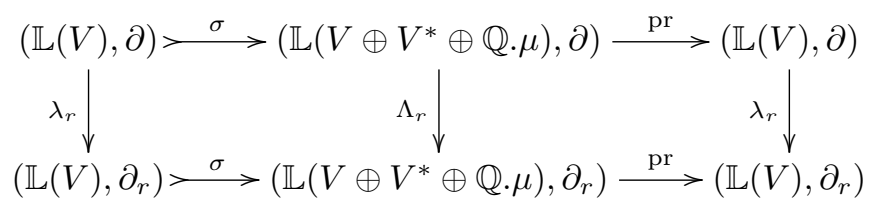

Par les considérations précédentes, il vient que, pour $r=3, \partial_{3}=\partial, \lambda_{3}=i d$ et $\Lambda_{3}=i d$ conviennent. Supposons avoir construit $\partial_{r}, \lambda_{r}, \Lambda_{r}, \ldots$ pour un certain $r \geqslant 3$. La relation de Jacobi permet d'écrire

$$
\epsilon_{r}=\sum_{i}\left[v_{i}, \alpha_{i}\right]+\sum_{i}\left[\gamma_{i}, v_{i}^{*}\right]+\sum_{i}\left[\beta_{i}, v_{i}^{*}\right]
$$

avec $\alpha_{i}, \gamma_{i} \in I \cap \mathbb{L}^{\geqslant r-1}\left(V \oplus V^{*}\right)$ et $\beta_{i} \in \mathbb{L}^{\geqslant r-1}(V)$. Comme $\sum_{i}\left[\gamma_{i}, v_{i}^{*}\right] \in[I, I]$, quitte à remplacer $\xi_{r}$ par $\xi_{r}+\sum_{i}\left[\gamma_{i}, v_{i}^{*}\right]$, nous pouvons supposer que $\gamma_{i}=0$. Ainsi

$$
\begin{aligned}
\partial_{r}(\mu) & =\sum_{i}\left[v_{i}, v_{i}^{*}\right]+\xi_{r}+\sum_{i}\left[v_{i}, \alpha_{i}\right]+\sum_{i}\left[\beta_{i}, v_{i}^{*}\right] \\
& =\sum_{i}\left[v_{i}+\beta_{i}, v_{i}^{*}+\alpha_{i}\right]-\sum_{i}\left[\beta_{i}, \alpha_{i}\right]+\xi_{r} .
\end{aligned}
$$

En posant $\phi\left(v_{i}\right)=v_{i}-\beta_{i}$ et $\Phi\left(v_{i}\right)=v_{i}-\beta_{i}, \Phi\left(v_{i}^{*}\right)=v_{i}^{*}-\alpha_{i}, \Phi(\mu)=\mu$ nous obtenons des isomorphismes

$$
\phi: \mathbb{L}(V) \stackrel{\cong}{\longrightarrow} \mathbb{L}(V) \quad \Phi: \mathbb{L}\left(V \oplus V^{*} \oplus \mu\right) \stackrel{\cong}{\longrightarrow} \mathbb{L}\left(V \oplus V^{*} \oplus \mu\right),
$$

qui commutent à l'inclusion $\sigma: \mathbb{L}(V) \longmapsto \mathbb{L}\left(V \oplus V^{*} \oplus \mathbb{Q} . \mu\right)$ et à la projection pr: $\mathbb{L}\left(V \oplus V^{*} \oplus \mathbb{Q} \cdot \mu\right) \rightarrow \mathbb{L}(V)$. Par conséquent, en posant $\partial_{r+1}:=\Phi \partial_{r} \Phi^{-1}$, nous obtenons sur $\mathbb{L}\left(V \oplus V^{*} \oplus \mathbb{Q} . \mu\right)$ une différentielle qui vérifie les propriétés 1 et 2 . Les isomorphismes $\lambda_{r+1}:=\phi \lambda_{r}$ et $\Lambda_{r+1}:=\Phi \Lambda_{r}$ satisfont automatiquement les propriétés requises. Il reste à vérifier la propriété 3 . Observons que $\Phi(I) \subset I$ et que $\Phi\left(\alpha_{i}\right)=\alpha_{i}+\alpha_{i}^{\prime}$ et $\Phi\left(\beta_{i}\right)=\beta_{i}+\beta_{i}^{\prime}$ avec $\alpha_{i}^{\prime} \in I \cap \mathbb{L} \geqslant r\left(V \oplus V^{*}\right)$ et 


$$
\begin{aligned}
& \beta_{i}^{\prime} \in \mathbb{L}^{\geqslant}\left(V \oplus V^{*}\right) \text {. Ainsi } \\
& \partial_{r+1}(\mu)=\Phi\left(\partial_{r}\left(\Phi^{-1}(\mu)\right)\right)=\Phi\left(\partial_{r}(\mu)\right) \\
& =\sum_{i}\left[v_{i}+\beta_{i}^{\prime}, v_{i}^{*}+\alpha_{i}^{\prime}\right]-\sum_{i}\left[\beta_{i}+\beta_{i}^{\prime}, \alpha_{i}+\alpha_{i}^{\prime}\right]+\Phi\left(\xi_{r}\right) \\
& =\sum_{i}\left[v_{i}, v_{i}^{*}\right]+\Phi\left(\xi_{r}\right) \\
& +\left\{\sum_{i}\left(\left[v_{i}, \alpha_{i}^{\prime}\right]+\left[\beta_{i}^{\prime}, v_{i}^{*}\right]+\left[\beta_{i}^{\prime}, \alpha_{i}^{\prime}\right]-\left[\beta_{i}+\beta_{i}^{\prime}, \alpha_{i}+\alpha_{i}^{\prime}\right]\right)\right\} .
\end{aligned}
$$

Posons $\xi_{r+1}=\Phi\left(\xi_{r}\right)$ et notons $\epsilon_{r+1}$ le terme entre accolades dans (5). Une vérification immédiate montre que $\xi_{r+1} \in[I, I]$ et que $\epsilon_{r+1} \in I \cap \mathbb{L}^{\geqslant r+1}\left(V \oplus V^{*}\right)$. Ceci termine la récurrence.

Pour $r=n$ nous avons $\epsilon_{n}=0$ pour des raisons de degrés. Il vient donc que $\left(\mathbb{L}\left(V \oplus V^{*} \oplus \mathbb{Q} \cdot \mu\right), \partial_{n}\right)$ est un modèle quasi-étrusque et que le diagramme

$$
\left(\mathbb{L}(V), \partial_{n}\right) \stackrel{\sigma}{\longrightarrow}^{\longrightarrow}\left(\mathbb{L}\left(V \oplus V^{*} \oplus \mathbb{Q} \cdot \mu\right), \partial_{n}\right) \stackrel{\operatorname{pr}}{\longrightarrow}\left(\mathbb{L}(V), \partial_{n}\right),
$$

est un modèle de (4).

\section{Modèle étrusque}

Étant donné un modèle de Quillen $L$, dont l'espace des indécomposables $Q L$ est de dimension finie, nous montrons dans cette section qu'il existe un et un seul modèle étrusque de socle $L$ et de dimension formelle $n$. Ce modèle, noté $\mathcal{B}^{n}(L)$, sera explicitement décrit.

\subsection{Unicité des modèles étrusques de socle donné}

Pour prouver qu'il existe au plus un modèle étrusque de socle $L$ et de dimension formelle $n$, nous aurons besoin du lemme suivant:

Lemme 6.1. Soient $Z=V \oplus Y$ une décomposition d'espaces vectoriels gradués de dimension finie, $\left\{v_{i}\right\}_{1 \leqslant i \leqslant n}$ une base homogène de $V, J=(Y)$ l'idéal dans $\mathbb{L}(Z)$ engendré par $Y$ et $\left\{\gamma_{i}\right\}_{1 \leqslant i \leqslant n}$ une famille de $J$. Si $\sum_{i}\left[v_{i}, \gamma_{i}\right]=0$ dans $\mathbb{L}(Z)$ alors $\forall i: \gamma_{i}=0$.

Preuve. Posons $\mathcal{B}_{0}=\{1\}$ et $\mathcal{B}_{l}=\left\{v_{i_{1}} \otimes \cdots \otimes v_{i_{l}} \mid 1 \leqslant i_{1}, \ldots, i_{l} \leqslant n\right\}$ de sorte que $\mathcal{B}:=\cup_{l \geqslant 0} \mathcal{B}_{l}$ est une base en monômes de $T(V)$. Posons

$$
\Gamma_{r}=T(V) \otimes\left(\otimes_{i=1}^{r}(Y \otimes T(V))\right) .
$$

Ainsi $\Gamma:=\oplus_{r} \geqslant 1 \Gamma_{r}$ est l'idéal engendré par $Y$ dans $T(Z)$. Donc $\gamma_{i} \in \Gamma$ et nous pouvons écrire $\gamma_{i}=\sum_{r \geqslant 1} \gamma_{i}^{(r)}$ avec $\gamma_{i}^{(r)} \in \Gamma_{r}$. L'équation $\sum_{i}\left[v_{i}, \gamma_{i}\right]=0$ entraîne que $\sum_{i}\left[v_{i}, \gamma_{i}^{(r)}\right]=0$ pour tout $r \geqslant 1$. Sans perte de généralité nous pouvons donc supposer que $\gamma_{i} \in \Gamma_{r}$ avec $r \geqslant 1$ fixé. Soit $\mathcal{Y}=\left\{y_{j}\right\}$ une base de $Y$. Posons $\mathcal{G}_{-1}=\emptyset$ et, 
pour $l \geqslant 0$,

$$
\mathcal{G}_{l}:=\left\{\beta_{0} \eta_{1} \beta_{1} \eta_{2} \ldots \eta_{r} \beta_{r} \mid \beta_{0} \in \mathcal{B}_{l}, \beta_{1}, \ldots, \beta_{r} \in \mathcal{B}, \eta_{1}, \ldots, \eta_{r} \in \mathcal{Y}\right\}
$$

de sorte que $\mathcal{G}:=\cup_{l} \mathcal{G}_{l}$ est une base de $\Gamma_{r}$. Par conséquent, il existe des $C_{i}(\xi) \in \mathbb{Q}$ tels que

$$
\gamma_{i}=\sum_{\xi \in \mathcal{G}} C_{i}(\xi) \cdot \xi
$$

Dès lors

$$
\begin{aligned}
0 & =\sum_{i}\left[v_{i}, \gamma_{i}\right] \\
& =\sum_{\xi \in \mathcal{G}} \sum_{i}\left(C_{i}(\xi) v_{i} \xi- \pm C_{i}(\xi) \xi v_{i}\right) \\
& =\sum_{l=0}^{\infty}\left\{\sum_{i} \sum_{\xi \in \mathcal{G}_{l-1}} C_{i}(\xi) v_{i} \xi-\sum_{i} \sum_{\xi \in \mathcal{G}_{l}} \pm C_{i}(\xi) \xi v_{i}\right\} .
\end{aligned}
$$

Remarquons que si $\xi \in \mathcal{G}_{l}$ alors $v_{i} \xi \in \mathcal{G}_{l+1}$ et $\xi v_{i} \in \mathcal{G}_{l}$. Comme

$$
\Gamma_{r}=\oplus_{l=0}^{\infty} T^{l}(V) \otimes\left(\otimes_{j=1}^{r}(Y \otimes T(V))\right),
$$

et comme $\left(v_{i} \cdot \mathcal{G}_{l-1}\right) \cup\left(\mathcal{G}_{l} \cdot v_{i}\right) \subset T^{l}(V) \otimes\left(\otimes_{j=1}^{r}(Y \otimes T(V))\right)$ il est clair que chacun des termes

$$
\sigma_{l}:=\left\{\sum_{i} \sum_{\xi \in \mathcal{G}_{l-1}} C_{i}(\xi) v_{i} \xi-\sum_{i} \sum_{\xi \in \mathcal{G}_{l}} \pm C_{i}(\xi) \xi v_{i}\right\},
$$

entre accolades dans la somme (6) doit être nul.

Utilisant le fait que $\sigma_{l}=0$, prouvons par récurrence sur $l$ que $C_{i}(\xi)=0$ pour tout $\xi \in \mathcal{G}_{l}$. Si $l=-1$ c'est évident puisque $\mathcal{G}_{-1}=\emptyset$. Supposons que $C_{i}\left(\mathcal{G}_{l-1}\right)=0$ pour un certain $l \geqslant 0$. Cette hypothèse de récurrence entraîne que le terme de gauche de la différence qui définit $\sigma_{l}$ est nul. Il en est donc de même du terme de droite:

$$
\sum_{i} \sum_{\xi \in \mathcal{G}_{l}} \pm C_{i}(\xi) \xi v_{i}=0
$$

Or $\left\{\xi v_{i} \mid \xi \in \mathcal{G}_{l}, 1 \leqslant i \leqslant n\right\}$ est une famille libre de $T(V \oplus Y)$ et donc tous les coefficients $C_{i}(\xi)$ sont nuls pour $\xi \in \mathcal{G}_{l}$. Ceci achève de démontrer que $\gamma_{i}=0$.

Établissons à présent l'unicité des modèles étrusques de socle et de dimension donnés:

Proposition 6.2. Deux modèles étrusques de même dimension formelle et admettant des socles isomorphes sont isomorphes.

Remarque. Nous démontrerons à la proposition 6.6 qu'on peut remplacer "isomorphe" par "quasi-isomorphe" dans l'énoncé précédent.

Preuve. Soient $L=\left(\mathbb{L}\left(V \oplus V^{*} \oplus \mathbb{Q} \cdot \mu\right), \partial\right)$ et $M=\left(\mathbb{L}\left(W \oplus W^{*} \oplus \mathbb{Q} \cdot \omega\right), \delta\right)$ deux modèles étrusques tels que $(\mathbb{L}(V), \partial)$ et $(\mathbb{L}(W), \delta)$ sont isomorphes et $\operatorname{deg}(\mu)=$ 
$\operatorname{deg}(\omega)$. Nous allons montrer que $L$ et $M$ sont isomorphes. Soient $\left\{v_{i}, v_{i}^{*}, \mu\right\}_{1 \leqslant i \leqslant l}$, $\left\{w_{i}, w_{i}^{*}, \omega\right\}_{1 \leqslant i \leqslant l}$ des bases adaptées de ces modèles étrusques. Comme les socles sont isomorphes, ces bases ont même longueur et sont dans les mêmes degrés. Notons encore $J=\left(W^{*}\right)$ l'idéal dans $M$ engendré par $W^{*}$.

Soit $\phi:(\mathbb{L}(V), \partial) \cong(\mathbb{L}(W), \delta)$ un isomorphisme entre les socles. Posons $\hat{v}_{i}=$ $\phi\left(v_{i}\right) \in \mathbb{L}(W)$ et $\hat{V}=\left\langle\hat{v}_{i}: 1 \leqslant i \leqslant l\right\rangle$ de sorte que $\mathbb{L}(\hat{V})=\mathbb{L}(W)$. Nous pouvons écrire $w_{j}=\sum_{i} q_{j}^{i} \hat{v}_{i}+\alpha_{j}$ avec $q_{j}^{i} \in \mathbb{Q}$ et $\alpha_{j} \in \mathbb{L}^{\geqslant 2}(\hat{V})$. La matrice $\left(q_{j}^{i}\right)_{1 \leqslant i, j \leqslant l}$ est inversible et notons $\left(r_{i}^{j}\right)_{1 \leqslant i, j \leqslant l}$ sa matrice inverse. Posons $\bar{w}_{i}^{*}:=\sum_{j} q_{j}^{i} w_{j}^{*} \in W^{*}$ de sorte que $w_{j}^{*}=\sum_{i} r_{i}^{j} \bar{w}_{i}^{*}$. Alors

$$
\begin{aligned}
\delta \omega & =\sum_{j}\left[w_{j}, w_{j}^{*}\right] \\
& =\sum_{j}\left[\sum_{i} q_{j}^{i} \hat{v}_{i}, w_{j}^{*}\right]+\sum_{j}\left[\alpha_{j}, \sum_{k} r_{k}^{j} \bar{w}_{k}^{*}\right] \\
& =\sum_{i}\left[\hat{v}_{i}, \sum_{j} q_{j}^{i} w_{j}^{*}\right]+\sum_{k}\left[\sum_{j} r_{k}^{j} \alpha_{j}, \bar{w}_{k}^{*}\right] \\
& =\sum_{i}\left[\hat{v}_{i}, \bar{w}_{i}^{*}\right]+\sum_{k}\left[\sum_{j} r_{k}^{j} \alpha_{j}, \bar{w}_{k}^{*}\right] .
\end{aligned}
$$

Comme $\alpha_{j} \in \mathbb{L} \geqslant 2(\hat{V}) \subset[M, M]$ et $W^{*} \cap \mathbb{L}(\hat{V})=0$, la relation de Jacobi permet de trouver $\operatorname{des} \alpha_{i}^{*} \in J \cap[M, M]$ tels que

$$
\sum_{k}\left[\left(\sum_{j} r_{k}^{j} \alpha_{j}\right), \bar{w}_{k}^{*}\right]=\sum_{i}\left[\hat{v}_{i}, \alpha_{i}^{*}\right] .
$$

Posons $\hat{v}_{i}^{*}=\bar{w}_{i}^{*}+\alpha_{i}^{*}$ de sorte que $\delta(\omega)=\sum_{i}\left[\hat{v}_{i}, \hat{v}_{i}^{*}\right]$ et posons $\hat{V}^{*}=\left\langle\hat{v}_{i}^{*}: 1 \leqslant i \leqslant l\right\rangle$.

Notons $\pi: M \rightarrow Q M=M /[M, M]$ la projection canonique sur les indécomposables de $M$. Il est clair que $\pi(\hat{V})=\pi(W)$ et $\pi\left(\hat{V}^{*}\right)=\pi\left(W^{*}\right)$. Donc $\mathbb{L}\left(\hat{V} \oplus \hat{V}^{*} \oplus \mathbb{Q} . \omega\right)=M$. De plus $\hat{V}^{*} \subset J$ d'où $\delta\left(\hat{V}^{*}\right) \subset J$.

Définissons un isomorphisme d'algèbres de Lie graduées

$$
\Phi: \mathbb{L}\left(V \oplus V^{*} \oplus \mathbb{Q} \cdot \mu\right) \rightarrow \mathbb{L}\left(W \oplus W^{*} \oplus \mathbb{Q} \cdot \omega\right),
$$

en posant $\Phi\left(v_{i}\right)=\hat{v}_{i}, \Phi\left(v_{i}^{*}\right)=\hat{v}_{i}^{*}$ et $\Phi(\mu)=\omega$. Montrons que $\Phi$ commute aux différentielles, $\Phi \circ \partial=\delta \circ \Phi$. Comme $\Phi \mid \mathbb{L}(V)=\phi$ est un morphisme différentiel nous savons déjà que $\Phi\left(\partial v_{i}\right)=\delta \Phi\left(v_{i}\right)$. Nous avons aussi

$$
\delta(\Phi(\mu))=\delta(\omega)=\sum_{i}\left[\hat{v}_{i}, \hat{v}_{i}^{*}\right]=\Phi(\partial \mu) .
$$

Enfin

$$
\begin{aligned}
0 & =\delta^{2}(\omega)-\Phi\left(\partial^{2}(\mu)\right) \\
& =\delta\left(\sum_{i}\left[\hat{v}_{i}, \hat{v}_{i}^{*}\right]\right)-\Phi\left(\partial\left(\sum_{i}\left[v_{i}, v_{i}^{*}\right]\right)\right)
\end{aligned}
$$


Homology, Homotopy and Applications, vol. 8(2), 2006

$$
\begin{aligned}
& =\sum_{i}\left(\left[\delta \hat{v}_{i}, \hat{v}_{i}^{*}\right]+(-1)^{\left|\hat{v}_{i}\right|}\left[\hat{v}_{i}, \delta \hat{v}_{i}^{*}\right]-\left[\Phi\left(\partial v_{i}\right), \Phi\left(v_{i}^{*}\right)\right]-(-1)^{\left|v_{i}\right|}\left[\Phi\left(v_{i}\right), \Phi\left(\partial v_{i}^{*}\right)\right]\right) \\
& =\sum_{i}(-1)^{\left|v_{i}\right|}\left[\hat{v}_{i}, \delta\left(\hat{v}_{i}^{*}\right)-\Phi\left(\partial v_{i}^{*}\right)\right] .
\end{aligned}
$$

En appliquant le lemme 6.1 à la famille $\gamma_{i}=\delta\left(\hat{v}_{i}^{*}\right)-\Phi\left(\partial v_{i}^{*}\right) \in J$ nous trouvons que $\delta\left(\hat{v}_{i}^{*}\right)=\Phi\left(\partial v_{i}^{*}\right)$ ce qui achève de prouver que $\delta \Phi=\Phi \partial$. Ainsi $\Phi$ est un isomorphisme de LGD entre $L$ et $M$.

\subsection{Construction $\mathcal{B}^{n}$}

Étant donné un modèle de Quillen $L$, dont l'espace des indécomposables est de dimension finie, nous construisons un modèle étrusque de socle $L$ et de dimension formelle $n$ que nous noterons $\mathcal{B}^{n}(L)$.

Dans cette construction, nous utiliserons le morphisme d'adjonction sur l'algèbre tensorielle $T(V)$ c'est-à-dire l'application linéaire

$$
\text { ad: } T(V) \otimes T(V) \rightarrow T(V), \alpha \otimes \beta \mapsto \operatorname{ad}(\alpha, \beta)
$$

définie par $\operatorname{ad}(1, \beta)=\beta$ et $\operatorname{ad}(\alpha \cdot v, \beta)=\operatorname{ad}(\alpha,[v, \beta])$ pour $\alpha, \beta \in T(V)$ et $v \in V$, le crochet dans $T(V)$ étant donné par $[\gamma, \beta]:=\gamma \cdot \beta-(-1)^{|\gamma||\beta|} \beta \cdot \gamma$.

Proposition 6.3. Le morphisme d'adjonction ad vérifie les propriétés suivantes:

(i) $\operatorname{ad}(T(V) \otimes \mathbb{L}(V)) \subset \mathbb{L}(V)$.

(ii) Si $\alpha, \alpha^{\prime}, \beta \in T(V)$ alors $\operatorname{ad}\left(\alpha, \operatorname{ad}\left(\alpha^{\prime}, \beta\right)\right)=\operatorname{ad}\left(\alpha \cdot \alpha^{\prime}, \beta\right)$.

(iii) Pour $\alpha \in \mathbb{L}(V)$ et $\beta \in T(V)$ homogènes on a $\operatorname{ad}(\alpha, \beta)=[\alpha, \beta]$.

(iv) Si $\delta$ est une dérivation de degré -1 sur $T(V)$ telle que $\delta(V) \subset \mathbb{L}(V)$ alors pour $\alpha, \beta \in T(V)$ homogènes on a

$$
\delta(\operatorname{ad}(\alpha, \beta))=\operatorname{ad}(\delta(\alpha), \beta)+(-1)^{|\alpha|} \operatorname{ad}(\alpha, \delta(\beta)) .
$$

Preuve. Les points $(i)$ et $(i i)$ sont des conséquences immédiates de la définition de ad. Le point ( $i i i)$ se montre aisément par récurrence sur la longueur en crochets de $\alpha$. Démontrons la formule $(i v)$. Si $\alpha=x_{1} \cdots x_{r}$ avec $x_{i} \in V$ alors:

$$
\begin{aligned}
\delta(\operatorname{ad}(\alpha, \beta))= & \delta\left(\left[x_{1},\left[\cdots,\left[x_{r}, \beta\right] \cdots\right]\right]\right) \\
= & \sum_{i}(-1)^{\left|x_{1}\right|+\cdots+\left|x_{i-1}\right|}\left[x_{1},\left[\cdots\left[\delta\left(x_{i}\right),\left[\cdots,\left[x_{r}, \beta\right]\right] \cdots\right]\right.\right. \\
& +(-1)^{|\alpha|}\left[x_{1},\left[\cdots,\left[x_{r}, \delta(\beta)\right] \cdots\right]\right],
\end{aligned}
$$

et en appliquant les points $(i i)$ et $(i)$ à cette dernière expression on obtient

$$
\begin{aligned}
\delta(\operatorname{ad}(\alpha, \beta))= & \sum_{i}(-1)^{\left|x_{1}\right|+\cdots+\left|x_{i-1}\right|} \operatorname{ad}\left(x_{1}, \cdots \operatorname{ad}\left(\delta\left(x_{i}\right), \cdots \operatorname{ad}\left(x_{r}, \beta\right)\right) \cdots\right) \\
& +(-1)^{|\alpha|} \operatorname{ad}\left(x_{1}, \cdots \operatorname{ad}\left(x_{r}, \delta(\beta)\right) \cdots\right) \\
= & \sum_{i}(-1)^{\left|x_{1}\right|+\cdots+\left|x_{i-1}\right|} \operatorname{ad}\left(x_{1} \cdots \delta\left(x_{i}\right) \cdots x_{r}, \beta\right) \\
& +(-1)^{|\alpha|} \operatorname{ad}\left(x_{1} \cdots x_{r}, \delta(\beta)\right)
\end{aligned}
$$


Homology, Homotopy and Applications, vol. 8(2), 2006

$$
=\operatorname{ad}(\delta(\alpha), \beta)+(-1)^{|\alpha|} \operatorname{ad}(\alpha, \delta(\beta)) .
$$

\subsection{Construction de $\mathcal{B}^{n}(L)$}

Soit $L=(\mathbb{L}(V), \partial)$ un modèle de Quillen dans lequel $V$ est un espace vectoriel gradué de dimension finie et supposons que $V_{i}=0$ pour $i \geqslant n-1$. Posons $V^{*}=$ $s^{n-2} \# V$, autrement dit, $\left(V^{*}\right)_{j}=\#\left(V_{n-2-j}\right)$. Choisissons une base homogène $\left\{v_{i}\right\}$ de $V$ et soit $\left\{v_{i}^{*}\right\}$ la base de $V^{*}$ caractérisée $\operatorname{par}\left\langle v_{j}, s^{(2-n)} v_{i}^{*}\right\rangle=\delta_{j}^{i}$ où $\delta_{j}^{i}$ est le symbole de Kronecker. Observons qu'il existe une unique famille $\left\{\gamma_{j}^{i}\right\} \subset T(V)$ telle que l'élément $\partial\left(v_{j}\right) \in \mathbb{L}(V) \subset T(V)$ s'écrit $\partial\left(v_{j}\right)=\sum_{i} v_{i} \cdot \gamma_{j}^{i}$. Posons

$$
\mathcal{B}^{n}(L):=\left(\mathbb{L}\left(V \oplus V^{*} \oplus \mathbb{Q} \cdot \mu\right), \delta\right),
$$

où $\operatorname{deg}(\mu)=n-1$ et $\delta$ est la dérivation d'algèbre de Lie définie par:

$$
\delta \mid V:=\partial, \quad \delta\left(v_{i}^{*}\right):=-(-1)^{\left|v_{i}\right|} \sum_{j} \operatorname{ad}\left(\gamma_{j}^{i}, v_{j}^{*}\right), \quad \delta(\mu):=\sum_{i}\left[v_{i}, v_{i}^{*}\right] .
$$

Avant de démontrer que $\mathcal{B}^{n}(L)$ est bien une algèbre de Lie différentielle, c'est-àdire que $\delta^{2}=0$, illustrons la construction par un exemple simple. Prenons

$$
L=\left(\mathbb{L}\left(x_{1}, y_{1}, z_{4}\right) ; \partial x=\partial y=0, \partial z=[x,[x, y]]\right)
$$

où les indices des générateurs indiquent leur degré. Comme $\partial z=x^{2} y-y x^{2}$ on a, pour $n \geqslant 6$,

$$
\mathcal{B}^{n}(L)=\left(\mathbb{L}\left(x, y, z, z_{n-6}^{*}, y_{n-3}^{*}, x_{n-3}^{*}, \mu_{n-1}\right), \delta\right),
$$

avec

$$
\begin{aligned}
\delta x & =\delta y=0 \\
\delta z & =[x,[x, y]] \\
\delta z^{*} & =0 \\
\delta y^{*} & =\operatorname{ad}\left(-x^{2}, z^{*}\right)=-\left[x,\left[x, z^{*}\right]\right] \\
\delta x^{*} & =\operatorname{ad}\left(x y, z^{*}\right)=\left[x,\left[y, z^{*}\right]\right] \\
\delta \mu & =\left[x, x^{*}\right]+\left[y, y^{*}\right]+\left[z, z^{*}\right] .
\end{aligned}
$$

Proposition 6.4. $\mathcal{B}^{n}(L)$ est une algèbre de Lie différentielle.

Preuve. Il suffit de prouver que $\delta^{2}=0$ sur les générateurs. Pour les générateurs $v_{i}$ c'est évident car $\delta \mid V=\partial$. Prouvons que $\delta^{2}(\mu)=0$. En utilisant la proposition 6.3 nous avons

$$
\begin{aligned}
\delta^{2}(\mu) & =\delta\left(\sum_{i}\left[v_{i}, v_{i}^{*}\right]\right) \\
& =\sum_{j}\left[\delta\left(v_{j}\right), v_{j}^{*}\right]+\sum_{i}(-1)^{\left|v_{i}\right|}\left[v_{i}, \delta\left(v_{i}^{*}\right)\right] \\
& =\sum_{j} \operatorname{ad}\left(\delta\left(v_{j}\right), v_{j}^{*}\right)+\sum_{i}(-1)^{\left|v_{i}\right|}\left[v_{i},-(-1)^{\left|v_{i}\right|} \sum_{j} \operatorname{ad}\left(\gamma_{j}^{i}, v_{j}^{*}\right)\right]
\end{aligned}
$$


Homology, Homotopy and Applications, vol. 8(2), 2006

$$
\begin{aligned}
& =\sum_{j} \operatorname{ad}\left(\sum_{i} v_{i} \gamma_{j}^{i}, v_{j}^{*}\right)-\sum_{i} \sum_{j} \operatorname{ad}\left(v_{i}, \operatorname{ad}\left(\gamma_{j}^{i}, v_{j}^{*}\right)\right) \\
& =0 .
\end{aligned}
$$

Avant de prouver que $\delta^{2}\left(v_{i}^{*}\right)=0$, nous établissons la relation suivante:

$$
(-1)^{\left|v_{i}\right|} \delta\left(\gamma_{k}^{i}\right)+\sum_{j} \gamma_{j}^{i} \cdot \gamma_{k}^{j}=0
$$

Fixons l'entier $k$. Nous avons

$$
\begin{aligned}
0=\delta^{2}\left(v_{k}\right) & =\delta\left(\sum_{i} v_{i} \gamma_{k}^{i}\right) \\
& =\sum_{j}\left(\delta\left(v_{j}\right)\right) \gamma_{k}^{j}+\sum_{i}(-1)^{\left|v_{i}\right|} v_{i}\left(\delta\left(\gamma_{k}^{i}\right)\right) \\
& =\sum_{j} \sum_{i} v_{i} \gamma_{j}^{i} \gamma_{k}^{j}+\sum_{i}(-1)^{\left|v_{i}\right|} v_{i}\left(\delta\left(\gamma_{k}^{i}\right)\right) \\
& =\sum_{i} v_{i}\left\{(-1)^{\left|v_{i}\right|} \delta\left(\gamma_{k}^{i}\right)+\sum_{j} \gamma_{j}^{i} \cdot \gamma_{k}^{j}\right\}
\end{aligned}
$$

Comme $T^{+}(V)=\oplus_{i} v_{i} \cdot T(V)$ la relation (8) est une conséquence de la nullité de cette dernière expression.

Montrons, enfin, que $\delta^{2}\left(v_{i}^{*}\right)=0$ :

$$
\begin{aligned}
\delta^{2}\left(v_{i}^{*}\right)= & (-1)^{\left|v_{i}\right|-1} \sum_{j} \delta\left(\operatorname{ad}\left(\gamma_{j}^{i}, v_{j}^{*}\right)\right) \\
= & (-1)^{\left|v_{i}\right|-1}\left[\sum_{j} \operatorname{ad}\left(\delta\left(\gamma_{j}^{i}\right), v_{j}^{*}\right)+\sum_{j}(-1)^{\left|\gamma_{j}^{i}\right|} \operatorname{ad}\left(\gamma_{j}^{i}, \delta\left(v_{j}^{*}\right)\right)\right] \\
= & \left.(-1)^{\left|v_{i}\right|-1}\right] \\
& {\left[\sum_{k} \operatorname{ad}\left(\delta\left(\gamma_{k}^{i}\right), v_{k}^{*}\right)+\sum_{j}(-1)^{\left|\gamma_{j}^{i}\right|} \operatorname{ad}\left(\gamma_{j}^{i},(-1)^{\left|v_{j}\right|-1} \sum_{k} \operatorname{ad}\left(\gamma_{k}^{j}, v_{k}^{*}\right)\right)\right] } \\
= & (-1)^{\left|v_{i}\right|-1} \sum_{k} \operatorname{ad}\left(\delta\left(\gamma_{k}^{i}\right)+\sum_{j}(-1)^{\left|\gamma_{j}^{i}\right|}(-1)^{\left|v_{j}\right|-1} \gamma_{j}^{i} \cdot \gamma_{k}^{j}, v_{k}^{*}\right) .
\end{aligned}
$$

En observant que

$$
\begin{aligned}
(-1)^{\left|\gamma_{j}^{i}\right|}(-1)^{\left|v_{j}\right|-1} & =(-1)^{\left|v_{i}\right|+\left|v_{i}\right|+\left|\gamma_{j}^{i}\right|+\left|v_{j}\right|-1} \\
& =(-1)^{\left|v_{i}\right|+\left|\delta\left(v_{j}\right)\right|+\left|\delta\left(v_{j}\right)\right|}=(-1)^{\left|v_{i}\right|}
\end{aligned}
$$

il vient

$$
\delta^{2}\left(v_{i}^{*}\right)=(-1)^{\left|v_{i}\right|-1} \sum_{k} \operatorname{ad}\left(\delta\left(\gamma_{k}^{i}\right)+\sum_{j}(-1)^{\left|v_{i}\right|} \gamma_{j}^{i} \cdot \gamma_{k}^{j}, v_{k}^{*}\right)
$$


et cette dernière expression est nulle par la relation (8).

Le modèle $\mathcal{B}^{n}(L)$ étant par construction un modèle étrusque, le résultat d'unicité de la Proposition 6.2 et la proposition précédente permettent d'établir l'énoncé suivant:

Théorème 6.5. Soit $L=(\mathbb{L}(V), \partial)$ un modèle de Quillen dans lequel $V$ est un espace vectoriel gradué de dimension finie et soit $n$ un entier tel que $V_{\geqslant n-1}=0$. Alors $\mathcal{B}^{n}(L)$ est l'unique modèle étrusque de socle $L$ et de dimension formelle $n$. De plus $\mathcal{B}^{n}(L)$ est indépendant du choix des indécomposables $V \subset L$.

La construction $\mathcal{B}^{n}(-)$ n'est pas fonctorielle mais elle préserve le type d'homotopie faible:

Proposition 6.6. Deux modèles étrusques de même dimension formelle et admettant des socles quasi-isomorphes sont quasi-isomorphes.

\section{Preuve de la proposition 6.6.}

Soit $L$ un modèle de Quillen tel que $Q L$ est de dimension finie et soit $L_{0}$ un modèle minimal de $L$. Grâce à la Proposition 6.2, il suffit de montrer que les modèles étrusques $\mathcal{B}^{n}(L)$ et $\mathcal{B}^{n}\left(L_{0}\right)$ sont quasi-isomorphes.

D'après [1, II.4.7], $L$ est isomorphe au produit libre du modèle de Quillen minimal $L_{0}$ et d'un modèle de Quillen contractile:

$$
L \cong L_{0} \coprod(\mathbb{L}(T, \partial T), \partial)
$$

où $\partial: T \rightarrow \partial T$ est un isomorphisme. Supposons que $L_{0}=(\mathbb{L}(V), \partial)$. Alors

$$
\mathcal{B}^{n}\left(L_{0}\right)=\left(\mathbb{L}\left(V \oplus V^{*} \oplus \mu\right), \delta\right),
$$

avec $\delta(\mu)=\sum_{i=1}^{l}\left[v_{i}, v_{i}^{*}\right]$ où $\left\{v_{i}\right\}$ et $\left\{v_{i}^{*}\right\}$ sont des bases de $V$ et $V^{*}$. Soit $\left\{t_{k}\right\}$ une base homogène de $T$. Soient $\left\{u_{k}\right\}:=\left\{\partial t_{k}\right\},\left\{t_{k}^{*}\right\}$ et $\left\{u_{k}^{*}\right\}$ les bases correspondantes de $\partial T, T^{*}:=s^{n-2} \# T$ et $(\partial T)^{*}:=s^{n-2} \#(\partial T)$. Alors

$$
\mathcal{B}^{n}(L)=\left(\mathbb{L}\left(V \oplus T \oplus \partial T \oplus V^{*} \oplus T^{*} \oplus(\partial T)^{*} \oplus \mathbb{Q} \cdot \omega\right), \hat{\delta}\right),
$$

avec $\hat{\delta}\left(v_{k}\right)=\delta\left(v_{k}\right), \hat{\delta}\left(t_{k}\right)=u_{k}, \hat{\delta}\left(u_{k}\right)=0, \hat{\delta}\left(v_{k}^{*}\right)=\delta\left(v_{k}^{*}\right), \hat{\delta}\left(u_{k}^{*}\right)=(-1)^{\left|t_{k}\right|} t_{k}^{*}, \hat{\delta}\left(t_{k}^{*}\right)=$ 0 et $\hat{\delta}(\omega)=\sum\left[v_{i}, v_{i}^{*}\right]+\sum\left[u_{k}, u_{k}^{*}\right]+\sum\left[t_{k}, t_{k}^{*}\right]$. Définissons un morphisme de LGD

$$
\phi: \mathcal{B}^{n}(\mathbb{L}(V), \partial) \rightarrow \mathcal{B}^{n}(\mathbb{L}(V \oplus \partial T \oplus T), \partial)
$$

$\operatorname{par} \phi\left(v_{i}\right)=v_{i}, \phi\left(v_{i}^{*}\right)=v_{i}^{*}$ et $\phi(\mu)=\omega-\sum_{k}\left[t_{k}, u_{k}^{*}\right]$. Un calcul montre que $\phi$ commute aux différentielles. D'autre part $\phi$ induit un quasi-isomorphisme entre les indécomposables, donc $\phi$ lui-même est un quasi-isomorphisme.

Remarque. Il existe des modèles de Quillen quasi-étrusques de même socle et de même dimension formelle qui ne sont pas quasi-isomorphes. En voici un exemple: considérons les modèles de Quillen $(\mathbb{L}(W), \delta)=\left(\mathbb{L}\left(u, v, v^{*}, u^{*}, \mu\right), \delta\right)$ et $(\mathbb{L}(\tilde{W}), \tilde{\delta})=$ $\left(\mathbb{L}\left(u, v, \tilde{v}^{*}, \tilde{u}^{*}, \tilde{\mu}\right), \tilde{\delta}\right)$ dont les générateurs correspondent aux tables ci-dessous. 


\begin{tabular}{|c|c|c|c|c|c|c|c|c|}
\hline gén & $u$ & $v$ & $v^{*}$ & \multicolumn{2}{|r|}{$u^{*}$} & \multicolumn{3}{|c|}{$\mu$} \\
\hline deg & 3 & 7 & 3 & \multicolumn{2}{|r|}{7} & \multicolumn{3}{|c|}{11} \\
\hline$\delta$ & 0 & $\frac{1}{2}[u, u]$ & 0 & {$\left[u, v^{*}\right]$} & $+\left[v^{*}, v^{*}\right.$ & {$\left[u, u^{*}\right]$} & $+\left[v, v^{*}\right]$ & $+2\left[u^{*}, v^{*}\right.$ \\
\hline gén & $u$ & $v$ & $\tilde{v}^{*}$ & $\tilde{u}^{*}$ & \multicolumn{2}{|c|}{$\tilde{\mu}$} & & \\
\hline deg & 3 & 7 & 3 & 7 & \multicolumn{2}{|c|}{11} & & \\
\hline$\tilde{\delta}$ & 0 & $\frac{1}{2}[u, u]$ & 0 & {$\left[u, \tilde{v}^{*}\right]$} & {$\left[u, \tilde{u}^{*}\right]$} & {$\left[v, \tilde{v}^{*}\right]$} & & \\
\hline
\end{tabular}

Ces deux modèles $(\mathbb{L}(W), \delta)$ et $(\mathbb{L}(\tilde{W}), \tilde{\delta})$ sont des modèles quasi-étrusques de socle $(\mathbb{L}(V), d)=\left(\mathbb{L}\left(u_{3}, v_{7}\right), d u_{3}=0, d v_{7}=\frac{1}{2}\left[u_{3}, u_{3}\right]\right)$ qui ne sont pas quasi-isomorphes. En effet s'ils étaient quasi-isomorphes, ils seraient isomorphes car ils sont minimaux. Or tout isomorphisme d'algèbres de Lie graduées $\phi: \mathbb{L}(W) \rightarrow \mathbb{L}(\tilde{W})$ vérifie $\phi(W) \subset \tilde{W}$ pour des raisons de degrés. Un calcul évident montre qu'un tel isomorphisme ne peut être compatible aux différentielles $\delta$ et $\tilde{\delta}$. Notons aussi que $(\mathbb{L}(W), \delta)$ ne peut être isomorphe à un modèle étrusque. En effet, ce serait un modèle étrusque de dimension formelle 12 et de socle isomorphe à $(\mathbb{L}(V), d)$. Par unicité, ce modèle étrusque devrait être isomorphe à $\mathcal{B}^{12}(\mathbb{L}(V), d)=(\mathbb{L}(\tilde{W}), \tilde{\delta})$. Ainsi $(\mathbb{L}(W), \delta)$ et $(\mathbb{L}(\tilde{W}), \tilde{\delta})$ seraient isomorphes ce qui, comme on vient de le voir, n'est pas le cas.

\section{Modèle de Quillen d'un bord homotopique de classe $\mathcal{S}$}

Soit $X$ un CW-complexe fini simplement connexe. Rappelons qu'un bord homotopique $\partial N$ de $X$ est dit de classe $\mathcal{S}$ s'il est la suspension d'un bord homotopique à section $\partial N^{\prime}$ de $X$, ce qui signifie que $\partial N$ est le double-mapping cylindre de l'inclusion $\partial N^{\prime} \hookrightarrow N^{\prime}$. Le but de cette section est d'établir le théorème suivant:

Théorème 7.1. Soient $X$ un $C W$-complexe fini simplement connexe et $L$ un modèle de Quillen de $X$ dont l'espace des indécomposables est de dimension finie. Si $n$ est un entier tel que $(Q L)_{\geqslant n-1}=0$, alors $\mathcal{B}^{n}(L)$ est un modèle de Quillen de tout bord homotopique de $X$ de classe $\mathcal{S}$ et de dimension $n$.

La preuve, qui se trouve dans la section 7.2, s'appuiera de manière essentielle sur un résultat général concernant le modèle du double mapping cylindre d'une rétraction établi dans la section 7.1.

Le théorème 7.1 permet d'étendre aux bords homotopiques de classe $\mathcal{S}$ le résultat d'unicité $[\mathbf{4}$, Theorem 6] du type d'homotopie rationnelle des bords homotopiques superstables:

Corollaire 7.2. Soit $X$ un $C W$-complexe fini simplement connexe. Alors le type d'homotopie rationnelle d'un bord homotopique de $X$ de classe $\mathcal{S}$ est complètement déterminé par sa dimension et par le type d'homotopie rationnelle de $X$.

\section{Remarques.}

1. Le résultat d'unicité du type d'homotopie rationnelle des bords homotopiques superstables demontré dans [4] s'appuie sur la construction d'un modèle d'algèbre graduée différentielle commutative (AGDC) des bords homotopiques de $X$ à partir d'un modèle d'AGDC $(A, d)$ de l'espace $X$. Si $(A, d)$ est une AGDC 1-connexe 
telle que $A^{>k}=0$ pour un certain entier positif $k$, alors tout bord homotopique de $X$ de dimension $n \geqslant 2 k+1$ admet comme modèle une AGDC, que nous noterons ici $B^{n}(A, d)$, construite à partir de $(A, d)$. En appliquant le foncteur de Quillen $L_{*}:\{$ AGDC connexes de type fini $\} \rightarrow\{$ LGD $\}$ (cf. $[\mathbf{1 0}$, I.1(7)]) à cette construction, nous obtenons un modèle de Quillen des bords homotopiques de $X$ de dimension $n$. Il est en fait possible de prouver que ce modèle est exactement $\mathcal{B}^{n}\left(L_{*}(A, d)\right)$, c'est-à-dire $\mathcal{B}^{n}\left(L_{*}(A, d)\right)=L_{*}\left(B^{n}(A, d)\right)$.

2. Nous voudrions ici observer que la construction $\mathcal{B}^{n}(L)$ développée dans ce travail pourrait guider l'étude des bords homotopiques en homotopie entière. Considérons le cas particulier suivant: $L$ est un modèle de Quillen de la forme

$$
L=(\mathbb{L}(U \oplus V), \partial) \quad \partial(U)=0 \quad \partial(V) \subset \mathbb{L}(U)
$$

et $n$ est un entier tel $U_{i} \oplus V_{i}=0$ pour $i \geqslant n-1$. On peut alors observer que dans le modèle de Quillen

$$
\mathcal{B}^{n}(L)=\left(\mathbb{L}\left(U \oplus V \oplus U^{*} \oplus V^{*} \oplus \mathbb{Q} \cdot \mu\right), \delta\right)
$$

la différentielle $\delta$ vérifie $\delta\left(V^{*}\right)=0$ et $\delta\left(U^{*}\right) \subset \mathbb{L}\left(U \oplus V^{*}\right)$. Autrement dit, ce modèle s'écrit

$$
\mathcal{B}^{n}(L)=\left(\mathbb{L}\left(\left(U \oplus V^{*}\right) \oplus\left(V \oplus U^{*}\right) \oplus \mathbb{Q} \cdot \mu\right), \delta\right)
$$

avec $\delta\left(U \oplus V^{*}\right)=0, \delta\left(V \oplus U^{*}\right) \subset \mathbb{L}\left(U \oplus V^{*}\right)$ et $\delta(\mu) \in \mathbb{L}\left(\left(U \oplus V^{*}\right) \oplus\left(V \oplus U^{*}\right)\right)$.

Un modèle de la forme (9) est typiquement le modèle de la cofibre homotopique d'une application $f: \Sigma A \rightarrow \Sigma B$ entre deux suspensions (on peut dans ce cas prendre $U=\tilde{H}_{*}(B), V=s \tilde{H}_{*}(A)$ et construire $\partial$ à partir de l'adjointe de $f$ et de l'homomorphisme de Hurewicz). Pour $n$ assez grand (de sorte, par exemple, à être dans le rang superstable) il suit du théorème 7.1 que $\mathcal{B}^{n}(L)$ est un modèle des bords homotopiques de dimension $n$ de $X:=\Sigma B \cup_{f} C \Sigma A$ et, en particulier, du bord homotopique trivial, i.e. associé au plongement de $X$ dans $\mathbb{R}^{n+1}$. Dans [5] il est prouvé que ce bord homotopique est homotopiquement équivalent à un espace de la forme

$$
Z=\left(\Sigma B \vee \Sigma A^{*}\right) \cup_{\Phi} C\left(\Sigma A \vee \Sigma B^{*}\right) \cup e^{n}
$$

Dans cet espace $A^{*}$ et $B^{*}$ sont des duaux de Spanier-Whitehead de $A$ et $B$ respectivement. La cohomologie réduite de ces espaces correspond donc aux espaces vectoriels $V^{*}$ et $U^{*}$. L'application d'attachement $\Phi$ est une version topologique de la différentielle $\delta$ de (10) et il est en fait possible d'établir une correspondance précise entre les différentes étapes de la construction de $\Phi$ décrites dans [5] et la formule définissant $\delta$. Dans [5], la construction de l'espace $Z$ a joué un rôle essentiel dans la détermination d'une condition suffisante au plongement du 2-cône $X$ dans un espace euclidien de codimension donnée. Dans la perpective d'étudier les plongements dans l'espace euclidien d'un espace obtenu par attachements successifs de $k \geqslant 3$ cônes, il serait très utile de pouvoir décrire le bord homotopique trivial de cet espace à travers une décomposition en cônes parallèle à celle de l'espace considéré (tout comme la décomposition de $Z$ est parallèle à celle de $X$ ). Nous espérons que la description explicite du modèle de Quillen de ce bord homotopique que nous avons ici obtenue pourra guider la construction d'une telle décomposition. 


\subsection{Modèle du double mapping cylindre d'une rétraction}

Soient $X$ et $Y$ deux espaces 1-connexes et $\xi: X \mapsto Y$ une cofibration admettant une rétraction $r: Y \rightarrow X$, c'est-à-dire $r \circ \xi=i d$. Nous noterons $I A$ le cylindre réduit d'un espace pointé $A$. Le double mapping cylindre (réduit) de $r$, noté $Z_{r, r}$, est obtenu comme push-out du diagramme

$$
X \vee X \stackrel{r \vee r}{<} Y \vee Y>\longrightarrow I Y .
$$

On a le diagramme

$$
X>\stackrel{\xi_{0}}{\longrightarrow} Z_{r, r} \stackrel{\bar{r}}{\longrightarrow} X,
$$

où $\xi_{0}$ est la cofibration $X \vee X \rightarrow Z_{r, r}$ précédée de l'inclusion du premier facteur et $\bar{r}$ est induite par l'application $I Y \rightarrow Y \stackrel{r}{\rightarrow} X$ et par la folding map $X \vee X \rightarrow X$. Notons que $\bar{r} \xi_{0}=i d$.

Par la Proposition 5.3, le diagramme

$$
X \stackrel{\xi}{\longmapsto} Y \stackrel{r}{\rightarrow} X
$$

admet un modèle de Quillen de la forme

$$
(\mathbb{L}(V), \partial) \stackrel{\sigma}{\longmapsto}(\mathbb{L}(V \oplus W), \partial) \stackrel{\operatorname{pr}}{\rightarrow}(\mathbb{L}(V), \partial),
$$

où $\sigma$ est une extension libre, pr est la projection évidente et $(\mathbb{L}(V), \partial)$ et $(\mathbb{L}(V \oplus W), \partial)$ sont des modèles minimaux.

L'espace $Y$ admet donc un modèle de Quillen minimal de la forme $(\mathbb{L}(V \oplus W), \partial)$ dans lequel la différentielle $\partial$ vérifie:

(i) $\partial(V) \subset \mathbb{L}(V)$;

(ii) $\partial(W)$ est inclus dans l'idéal $(W)$ engendré par $W$.

Écrivons la différentielle $\partial$ sous la forme $\partial=\partial_{0}+\partial_{+}$où $\partial_{0}$ est la partie de $\partial$ qui préserve la longueur en $W$ tandis que $\partial_{+}$est la partie qui l'augmente.

Considérons l'algèbre de Lie graduée $\mathbb{L}(V \oplus s W)$ et définissons

$$
S:[\mathbb{L}(V), W] \rightarrow \mathbb{L}(V \oplus s W)
$$

par $S[a, w]=(-1)^{|a|}[a, s w]$ pour $a \in \mathbb{L}(V)$ et $w \in W$.

Proposition 7.3. Soit $(\mathbb{L}(V), \partial) \longmapsto(\mathbb{L}(V \oplus W), \partial) \stackrel{\mathrm{pr}}{\rightarrow}(\mathbb{L}(V), \partial)$ un modèle de Quillen minimal de

$$
X \stackrel{\xi}{\longmapsto} Y \stackrel{r}{\rightarrow} X
$$

tel que $(\mathbb{L}(V), \partial)$ et $(\mathbb{L}(V \oplus W), \partial)$ sont des modèles minimaux. Alors

$$
(\mathbb{L}(V), \partial) \longmapsto(\mathbb{L}(V \oplus s W), \tilde{\partial}) \stackrel{\mathrm{pr}}{\rightarrow}(\mathbb{L}(V), \partial),
$$

où $\tilde{\partial}$ est donnée par $\tilde{\partial}_{\mid V}=\partial$ et $\tilde{\partial}(s w)=-S\left(\partial_{0} w\right)$, est un modèle du diagramme

$$
X \stackrel{\xi_{0}}{\longrightarrow} Z_{r, r} \stackrel{\bar{r}}{\longrightarrow} X .
$$


Preuve. Remarquons tout d'abord que $Z_{r, r}$ est homotopiquement équivalent au push-out du diagramme

$$
X<\sim \sim \sim Z_{r, r}
$$

où la cofibration $I X \longmapsto Z_{r, r}$ est induite, de manière évidente, par $\xi$. Si on écrase d'abord $I X$ sur $X$ dans $I Y$, pour obtenir l'espace $I_{X} Y$, on voit facilement que $Z_{r, r}$ est homotopiquement équivalent au push-out de

$$
X \leftarrow Y \vee_{X} Y \longmapsto I_{X} Y .
$$

Nous allons construire un modèle de $Z_{r, r}$ en construisant un modèle de ce dernier push-out puis nous montrerons que la différentielle de ce modèle a la forme annoncée.

1) Afin de construire un modèle de $Y \vee_{X} Y \rightarrow X$, on considère une copie $\hat{W}$ de $W$ ainsi que l'isomorphisme

$$
\widehat{\imath} \mathbb{L}(V \oplus W) \rightarrow \mathbb{L}(V \oplus \hat{W}),
$$

défini par $v \mapsto v$ et $w \mapsto \hat{w}$. L'algèbre de Lie $(\mathbb{L}(V \oplus W \oplus \hat{W}), \partial)$ où $\partial_{\mid V \oplus W}$ est la différentielle de $(\mathbb{L}(V \oplus W), \partial)$ et $\partial(\hat{w}):=\widehat{\partial w}$ est un modèle de $Y \vee_{X} Y$ et un modèle de $Y \vee_{X} Y \rightarrow X$ est alors donné par la projection

$$
(\mathbb{L}(V \oplus W \oplus \hat{W}), \partial) \stackrel{\mathrm{pr}}{\rightarrow}(\mathbb{L}(V), \partial) .
$$

À la manière de Tanré (cf. [10, II.5(1)]), on construit un modèle de $Y \vee_{X} Y \longmapsto$ $I_{X} Y$ comme suit:

On considère une autre copie $\bar{W}$ de $W$ et l'algèbre de Lie $(\mathbb{L}(V \oplus W \oplus \bar{W} \oplus$ $s W), D)$ où $D_{\mid V \oplus W}=\partial, D \bar{w}=0$ et $D s w=\bar{w}$. On munit $\mathbb{L}(V \oplus W \oplus \bar{W} \oplus s W)$ de la dérivation d'algèbre de Lie $i$ de degré +1 donnée par

$$
i(v)=0, i(w)=s w, i(\bar{w})=i(s w)=0
$$

puis on considère la dérivation $\theta=D i+i D$ de degré 0 . À cette dérivation (qui vérifie $\theta D=D \theta)$ est associé l'automorphisme $e^{\theta}:=\sum_{n \geqslant 0} \frac{\theta^{n}}{n !}$ de l'algèbre de Lie différentielle $(\mathbb{L}(V \oplus W \oplus \bar{W} \oplus s W), D)$. Un modèle de $Y \vee_{X} Y \hookrightarrow I_{X} Y$ est alors donné par le morphisme

$$
\phi:(\mathbb{L}(V \oplus W \oplus \hat{W}), \partial) \rightarrow(\mathbb{L}(V \oplus W \oplus \bar{W} \oplus s W), D),
$$

défini par $v \mapsto v, w \mapsto w$ et $\hat{w} \mapsto e^{\theta}(w)$.

Soit $g: \mathbb{L}(V \oplus W \oplus \hat{W} \oplus s W) \rightarrow \mathbb{L}(V \oplus W \oplus \bar{W} \oplus s W)$ défini par $g(v)=v$, $g(w)=w, g(\hat{w})=e^{\theta}(w)$ et $g(s w)=s w$. C'est un isomorphisme et $\phi$ admet la factorisation suivante

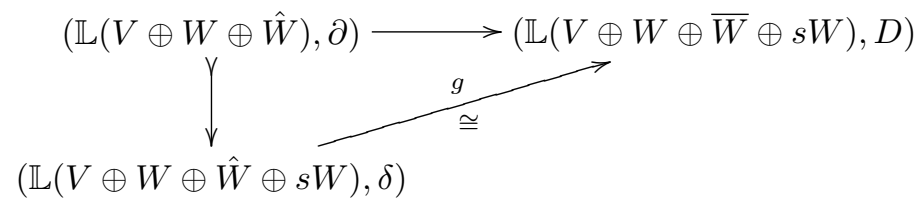

où la différentielle $\delta$ étend la différentielle $\partial$ et vérifie $\delta=g^{-1} D g$. 
Un modèle du double mapping cylindre de la rétraction $r: Y \rightarrow X$ est alors obtenu en formant le push-out de l'extension

$$
(\mathbb{L}(V \oplus W \oplus \hat{W}), \partial)>(\mathbb{L}(V \oplus W \oplus \hat{W} \oplus s W), \delta),
$$

le long de la projection

$$
(\mathbb{L}(V \oplus W \oplus \hat{W}), \partial) \stackrel{\mathrm{pr}}{\rightarrow}(\mathbb{L}(V), \partial) .
$$

Ce push-out est l'algèbre de Lie $\mathbb{L}(V \oplus s W)$ munie de la diferentielle pr $\delta$ où pr est la projection $\mathbb{L}(V \oplus W \oplus \hat{W} \oplus s W) \rightarrow \mathbb{L}(V \oplus s W)$.

2) Montrons à présent que la différentielle pr $\delta$ a la forme annoncée. Il est clair que $\tilde{\operatorname{pr}} \delta v=\partial v$. Il nous reste à prouver que $\tilde{\operatorname{pr}} \delta(s w)=-S\left(\partial_{0} w\right)$. Munissons

$$
\mathbb{L}(V \oplus W \oplus \hat{W} \oplus s W),
$$

des dérivations $h:=g^{-1} i g$ et $\rho:=g^{-1} \theta g(=\delta h+h \delta)$ de degré respectif +1 et 0 . À travers l'isomorphisme $g$ on calcule que

- $h(v)=h(s w)=0$ et $h(w)=s w$.

- $\rho(v)=\rho(s w)=0$.

- $h \delta s w=\rho \delta s w=0$.

- $e^{\rho}(w)=\hat{w}$.

Par conséquent:

$$
\delta s w=\delta h w=\rho w-h \delta w=\rho w-h \partial w=\rho w-h \partial_{0} w-h \partial_{+} w .
$$

Soit $J$ l'idéal de $\mathbb{L}(V \oplus W \oplus \hat{W} \oplus s W)$ engendré par $W \oplus \hat{W}$. Comme $\partial_{+} w \in[J, J]$ et $h$ est une dérivation, il est clair que $h\left(\partial_{+} w\right) \in J$ et, par suite, $\tilde{\operatorname{pr}} h\left(\partial_{+} w\right)=0$. Observons également que la restriction de pr $h$ à $[\mathbb{L}(V), W]$ est exactement l'application $S$. Par conséquent

$$
\begin{aligned}
\tilde{\operatorname{pr}} \delta s w & =\tilde{\operatorname{pr}} \rho w-\tilde{\operatorname{pr}} h \partial_{0} w-\tilde{\operatorname{pr}} h \partial_{+} w \\
& =\tilde{\operatorname{pr}} \rho w-S \partial_{0} w .
\end{aligned}
$$

Afin d'achever la preuve, il nous reste à prouver que $\rho w \in J$, ce que nous allons faire par récurrence sur le degré. Supposons que $|w|=n$ et que $\rho\left(W_{<n}\right) \in J$. De l'égalité $e^{\rho}(w)=\hat{w}$ vient

$$
\rho w=\hat{w}-w-\sum_{k \geqslant 2} \frac{\rho^{k}(w)}{k !} .
$$

Clairement $\hat{w}-w \in J$. Par ailleurs, pour tout $k \geqslant 2$, on a:

$$
\rho^{k}(w)=\rho^{k-1}(\rho w)=\rho^{k-1}(h \delta w+\delta h w)=\rho^{k-1}(h \partial w+\delta s w) .
$$

Comme $\rho \delta s w=0$, il vient (pour $k \geqslant 2$ ):

$$
\rho^{k}(w)=\rho^{k-1}(h \partial w) .
$$

Pour raison de degré, $\partial w \in \mathbb{L}\left(V \oplus W_{<n}\right)$ et, par suite,

$$
h \partial w \in \mathbb{L}\left(V \oplus W_{<n} \oplus s W_{<n}\right) .
$$

Par hypothèse de récurrence $\rho\left(V \oplus W_{<n} \oplus s W_{<n}\right) \subset J$. En utilisant la formule de 
Leibniz

$$
\rho^{p}[a, b]=\sum_{i=0}^{p}\left(\begin{array}{c}
p \\
i
\end{array}\right)\left[\rho^{i}(a), \rho^{p-i}(b)\right]
$$

on établit que $\rho^{p}\left(\mathbb{L}\left(V \oplus W_{<n} \oplus s W_{<n}\right)\right) \subset J$ pour tout $p \geqslant 1$. Cela montre que $\rho^{k-1}(h \partial w) \in J$ pour tout $k \geqslant 2$ et, par suite, que $\rho w \in J$ ce qui termine la récurrence.

\subsection{Preuve du théorème 7.1}

Nous utiliserons dans cette preuve les notations introduites en 7.1. Soit $L$ un modèle de Quillen de $X$ et soit $\partial N$ le bord d'un thickening $N$ de classe $\mathcal{S}$ de $X$. Par hypothèse $N$ est la suspension d'un thickening $\phi^{\prime}: X \stackrel{\simeq}{\rightarrow} N^{\prime}$ à section $\nu^{\prime}: X \rightarrow \partial N^{\prime}$ et $\partial N$ est alors le double-mapping cylindre de l'inclusion $j^{\prime}: \partial N^{\prime} \hookrightarrow N^{\prime}$. L'inclusion

$$
j: \partial N=\left(\partial N^{\prime} \times I\right) \cup_{\partial N^{\prime} \times\{0,1\}}\left(N^{\prime} \times\{0,1\}\right) \rightarrow N,
$$

admet comme section homotopique l'application $\nu: X \rightarrow \partial N$ donnée par $\nu(x)=$ $\left[\nu^{\prime}(x), 0\right]$. En remplaçant le diagramme $X \stackrel{\nu^{\prime}}{\rightarrow} \partial N^{\prime} \stackrel{j^{\prime}}{\hookrightarrow} N^{\prime}$ par un diagramme faiblement équivalent

$$
X \stackrel{\xi}{\longrightarrow} Y \stackrel{r}{\longrightarrow} X
$$

le diagramme $X \stackrel{\nu}{\rightarrow} \partial N \stackrel{j}{\hookrightarrow} N$ s'avère alors être faiblement équivalent au diagramme suivant:

$$
X \stackrel{\xi_{0}}{\longrightarrow} Z_{r, r} \stackrel{\bar{r}}{\longrightarrow} X .
$$

Or on sait par le théorème 5.2 que le diagramme $X \stackrel{\nu^{\prime}}{\rightarrow} \partial N^{\prime} \stackrel{j^{\prime}}{\hookrightarrow} N^{\prime}$ (et donc également $X \stackrel{\xi}{\longmapsto} Y \stackrel{r}{\rightarrow} X$ ) admet un modèle de Quillen de la forme

$$
(\mathbb{L}(V), \partial) \stackrel{\sigma}{\longrightarrow}\left(\mathbb{L}\left(V \oplus V^{*} \oplus \mathbb{Q} \cdot \mu\right), \partial\right) \stackrel{\mathrm{pr}}{\longrightarrow}(\mathbb{L}(V), \partial),
$$

où $\sigma$ est une extension libre, pr est la projection évidente, $(\mathbb{L}(V), \partial)$ est un modèle minimal de $X$ et le terme du milieu est un modèle quasi-étrusque minimal de $\partial N^{\prime}$. En écrivant la différentielle $\partial=\partial_{0}+\partial_{+}$et en appliquant la Proposition 7.3 nous obtenons que

$$
(\mathbb{L}(V), \partial) \longmapsto\left(\mathbb{L}\left(V \oplus s V^{*} \oplus \mathbb{Q} \cdot s \mu\right), \tilde{\partial}\right) \stackrel{\text { pr }}{\rightarrow}(\mathbb{L}(V), \partial),
$$

où $\tilde{\partial}$ est donnée par $\tilde{\partial}_{\mid V}=\partial, \tilde{\partial}\left(s v^{*}\right)=-S\left(\partial_{0} v^{*}\right)$ et $\tilde{\partial}(s \mu)=-S\left(\partial_{0} \mu\right)$ est un modèle du diagramme

$$
X \stackrel{\xi_{0}}{\longrightarrow} Z_{r, r} \stackrel{\bar{r}}{\longrightarrow} X .
$$

Montrons que $\left(\mathbb{L}\left(V \oplus s V^{*} \oplus \mathbb{Q} . s \mu\right), \tilde{\partial}\right)$ est étrusque. Par définition de $S$, il est clair que $\tilde{\partial}\left(s V^{*}\right)$ est inclus dans l'idéal $\left(s V^{*}\right)$ engendré par $s V^{*}$. Calculons $\tilde{\partial}(s \mu)$. Comme $\left(\mathbb{L}\left(V \oplus V^{*} \oplus \mathbb{Q} \cdot \mu\right), \partial\right)$ est quasi-étrusque, nous savons que $\partial(\mu)=\sum_{i=1}^{l}\left[v_{i}, v_{i}^{*}\right]+\epsilon$ 
où $\left\{v_{i}\right\}_{1 \leqslant i \leqslant l}$ et $\left\{v_{i}^{*}\right\}_{1 \leqslant i \leqslant l}$ sont des bases homogènes respectivement de $V$ et de $V^{*}$ et $\epsilon \in\left[\left(V^{*}\right),\left(V^{*}\right)\right]$. Par conséquent $\partial_{0}(\mu)=\sum_{i=1}^{l}\left[v_{i}, v_{i}^{*}\right]$ et il vient

$$
\tilde{\partial}(s \mu)=-\sum_{i=1}^{l} S\left(\left[v_{i}, v_{i}^{*}\right]\right)=-\sum_{i=1}^{l}(-1)^{\left|v_{i}\right|}\left[v_{i}, s v_{i}^{*}\right]=\sum_{i=1}^{l}\left[(-1)^{\left|v_{i}\right|+1} v_{i}, s v_{i}^{*}\right] .
$$

En remplaçant la base $\left\{v_{i}\right\}$ de $V$ par la base $\left\{(-1)^{\left|v_{i}\right|+1} v_{i}\right\}$ nous obtenons alors que $\left(\mathbb{L}\left(V \oplus s V^{*} \oplus \mathbb{Q} . s \mu\right), \tilde{\partial}\right)$ est un modèle étrusque de socle $(\mathbb{L}(V), \partial)$ et de dimension formelle $n$. Par le Théorème 6.5 ce modèle est $\mathcal{B}^{n}(\mathbb{L}(V), \partial)$. Finalement, par la Proposition 6.6, on obtient que $\mathcal{B}^{n}(L)$ est un modèle de $Z_{r, r}$ et donc de $\partial N$.

\section{Un exemple}

L'exemple suivant montre que dans les théorèmes 4.1 et 7.1 on ne peut pas affaiblir l'hypothèse que le bord homotopique $\partial N$ est de classe $\mathcal{S}$.

Proposition 8.1. Il existe un bord homotopique suspension (et donc à section) $\partial N$ qui n'admet pas de modèle de Quillen étrusque et pour lequel on n'a pas d'isomorphisme d'algèbre

$$
H^{*}(\partial N ; \mathbb{Q}) \cong H^{*}(N ; \mathbb{Q}) \oplus \# s^{n} H^{*}(N ; \mathbb{Q}),
$$

tel que $\left(\# s^{n} H^{*}(N ; \mathbb{Q})\right) .\left(\# s^{n} H^{*}(N ; \mathbb{Q})\right)=0$.

Nous démontrons d'abord deux lemmes. Dans la suite de cette section nous écrirons $H^{*}(-)$ pour $H^{*}(-; \mathbb{Q})$.

Lemme 8.2. Il existe un fibré vectoriel réel $\xi$ de rang 4 et de base $\mathbb{C P}(4)$

$$
\xi: \mathbb{R}^{4} \hookrightarrow E \xi \rightarrow \mathbb{C P}(4),
$$

dont la classe d'Euler $e(\xi) \in H^{4}(\mathbb{C P}(4))$ est le carré d'un élément non nul $x \in$ $H^{2}(\mathbb{C P}(4))$.

Preuve. Considérons le fibré de Hopf quaternionique

$$
\eta: \mathbb{H} \hookrightarrow E \eta \rightarrow S^{4},
$$

dont la classe d'Euler est non nulle et notons $f: S^{4} \rightarrow B S O(4)$ son application classifiante. Le fibré en sphère associé est

$$
S \eta: S^{3} \rightarrow S^{7} \rightarrow S^{4} .
$$

En passant à l'espace des orbites par l'action libre du cercle $S^{1}$ sur l'espace total et la fibre de ce fibré en sphères, nous obtenons un fibré

$$
\overline{S \eta}: S^{2} \longrightarrow \mathbb{C P}(3) \stackrel{p}{\longrightarrow} S^{4},
$$

et l'application $H^{*}(p)$ est injective. Soit $h \in \pi_{7}(\mathbb{C P}(3))$ l'application d'attachement de la dernière cellule de $\mathbb{C P}(4)$. Comme $\pi_{7}(B S O(4)) \otimes \mathbb{Q}=0$, il existe un entier 
$k \geqslant 1$ tel que $(k . f) \circ p \circ h$ est homotopiquement triviale. Nous en déduisons le diagramme suivant

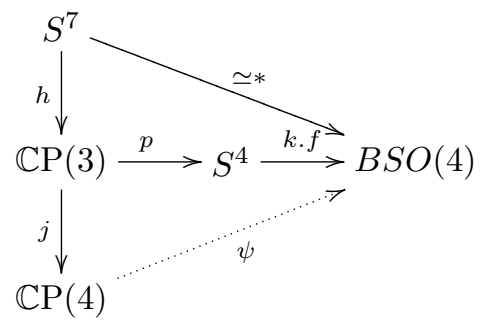

où $\psi$ est l'application induite sur la cofibre de $h$.

Notons $\gamma$ le fibré orienté universel de rang 4 sur $B S O(4)$ et $\xi:=\psi^{*}(\gamma)$ le fibré induit sur $\mathbb{C P}(4)$. Par la naturalité de la classe d'Euler,

$$
j^{*}(e(\xi))=p^{*}(k . e(\eta)) \neq 0 .
$$

Quitte à remplacer $k$ par un multiple non nul convenable, $j^{*}(e(\xi))$ est un carré non nul et il en est de même de $e(\xi)$.

Le fibré en disques associé au fibré $\xi$ du lemme précédent donne un thickening $N^{\prime}=D \xi$ de $\mathbb{C P}(4)$ et $\partial N^{\prime}=S \xi$ est un bord homotopique de $\mathbb{C P}(4)$. Considérons le thickening suspension $N=N^{\prime} \times[0,1]$.

Lemme 8.3. Toute classe de cohomologie non nulle $\alpha \in H^{4}(\partial N)$ vérifie $\alpha^{2} \neq 0$.

Preuve. Nous calculons la cohomologie de $\partial N$ à l'aide des modèles d'AGDC (voir $[3]$ pour la théorie). Un modèle d'AGDC de $\mathbb{C P}(4)$ est $H=\left(\mathbb{Q}\left[x_{2}\right] /\left(x^{5}\right), 0\right)$ et un modèle du fibré en sphère $S^{3} \hookrightarrow \partial N^{\prime} \rightarrow \mathbb{C P}(4)$ est

$$
H>\longrightarrow\left(H \otimes \wedge\left(u_{3}\right), d u=x^{2}\right) \longrightarrow(\wedge(u), 0) .
$$

Un modèle surjectif de $\partial N^{\prime} \hookrightarrow N^{\prime} \simeq \mathbb{C P}(4)$ est

$$
\left(H \otimes \wedge\left(u_{3}, \bar{u}_{4}\right), D u=x^{2}-\bar{u}, D \bar{u}=0\right) \stackrel{\pi}{\longrightarrow}\left(H \otimes \wedge\left(u_{3}\right), d\right),
$$

avec $\pi(\bar{u})=0, \pi(u)=u$ et $\pi \mid H=$ id. Comme $\partial N$ est homotopiquement équivalent à la somme amalgamée du diagramme $N^{\prime} \longleftrightarrow \partial N^{\prime} \longleftrightarrow N^{\prime}$, un modèle d'AGDC de $\partial N$ est donné par le produit fibré d'AGDC du diagramme

$$
\left(H \otimes \wedge\left(u_{3}, \bar{u}_{4}\right), D u=x^{2}-\bar{u}, D \bar{u}=0\right) \stackrel{\pi}{\longrightarrow}\left(H \otimes \wedge\left(u_{3}\right), d\right) \longleftrightarrow H .
$$

Ce produit fibré est $A=\left(H \otimes\left(\mathbb{Q} .1 \oplus\left(\wedge^{+} \bar{u} \otimes \wedge u\right)\right), \hat{D}\right)$ avec

$$
\begin{aligned}
\hat{D}\left(x^{i} \otimes \bar{u}^{k}\right) & =0, \\
\hat{D}\left(x^{i} \otimes \bar{u}^{k} \otimes u\right) & =x^{i+2} \otimes \bar{u}^{k}-x^{i} \otimes \bar{u}^{k+1} .
\end{aligned}
$$

Donc $H^{*}(\partial N)=H(A)=H \otimes\left\langle 1, \bar{u}_{4}\right\rangle$ où la structure d'algèbre est celle qui étend celle de $H$-module libre et qui vérifie $\bar{u}^{2}=x^{2} . \bar{u}$. Une base de $H^{4}(\partial N)$ est $\left\{x^{2}, \bar{u}\right\}$ et aucune combinaison linéaire non nulle de ces deux classes n'est de carré nul. 
Preuve de la Proposition 8.1. Considérons, comme précédemment, le thickening suspension $N=N^{\prime} \times[0,1]$ de $\mathbb{C P}(4)$ dans lequel $N^{\prime}$ est le fibré en disques associé au fibré $\xi$ du lemme 8.2. Le lemme 8.3 implique qu'il ne peut exister d'isomorphisme d'algèbre

$$
H^{*}(\partial N) \cong H^{*}(\mathbb{C P}(4)) \oplus \# s^{12} H^{*}(\mathbb{C P}(4)),
$$

tel que $\# s^{12} H^{*}(\mathbb{C P}(4))$. \# $s^{12} H^{*}(\mathbb{C P}(4))=0$ car $\# s^{12} H^{*}(\mathbb{C P}(4))$ contient un élément non nul de degré 4 et de carré nul. De même $\partial N$ ne peut admettre de modèle étrusque $\left(\mathbb{L}\left(V \oplus V^{*} \oplus \mathbb{Q} . \mu\right), \delta\right)=\mathcal{B}^{12}(\mathbb{L}(V), \partial)$. En effet l'interprétation des indécomposables $V^{*}$ comme sous-espace vectoriel de $H_{*}(\partial N)$ et la forme de la partie quadratique de $\delta$ qui doit vérifier $\delta_{2}\left(V^{*}\right) \subset\left[V, V^{*}\right]$ donnerait une classe de cohomologie non nulle de $H^{4}(\partial N)$ et de carré nul.

Remarque. Dans l'exemple de la proposition 8.1 la signature de $\partial N$ est non nulle, comme on le voit à l'aide du lemme 8.3. Une question intéressante soulevée par le rapporteur est celle de savoir si tout bord homotopique à section de signature nulle admet un modèle étrusque. Une réponse positive à cette question devrait donner une caractérisation complète des bords homotopiques admettant un tel modèle.

\section{References}

[1] H.J. Baues, J.-M. Lemaire Minimal models in homotopy theory. Math. Ann. 225:219-245, 1977.

[2] W. Browder Embedding 1-connected manifolds. Bull. Amer. Math. Soc. 72:225-231, 1966.

[3] Y. Félix, S. Halperin, J.-C. Thomas Rational homotopy theory. Graduate Text in Mathematics, Vol. 200, Springer-Velag, 2001.

[4] P. Lambrechts Cochain models of thickenings and applications to rational LScategory. Manuscripta Math. 103:143-160, 2000.

[5] P. Lambrechts, D. Stanley, L. Vandembroucq Embeddings up to homotopy of two-cones into Euclidean space. Trans. AMS 354:3973-4013, 2002.

[6] M. Majewski Rational homotopical models and uniqueness. Mem. Amer. Math. Soc. 143, No. 682, 2000.

[7] D. Quillen Rational homotopy theory. Annals Math. 90:205-295, 1969.

[8] C.P. Rourke, B.J. Sanderson Introduction to Piecewise-Linear Topology. Ergebnisse der Mathematik und ihrer Grenzgebiete, Band 69. Springer-Verlag, 1972.

[9] J. Stasheff Rational Poincaré duality spaces. Illinois J. Math. 27:104-109, 1983.

[10] D. Tanré Homotopie rationelle: modèles de Chen, Quillen, Sullivan. Lecture Notes in Mathematics, Vol. 1025, Springer-Verlag, Berlin, 1983.

[11] C.T.C. Wall Classification problems in differential topology IV: thickenings. Topology 5:73-94, 1966. 
Thomas Kahl kahl@math.uminho.pt

Centro de Matemática

Universidade do Minho

Campus de Gualtar

4710-057 Braga

Portugal

Pascal Lambrechts lambrechts@math.ucl.ac.be

Université Catholique de Louvain

Département de Mathématique

2, chemin du cyclotron

1348 Louvain-la-Neuve

Belgium

Lucile Vandembroucq lucile@math.uminho.pt

Centro de Matemática

Universidade do Minho

Campus de Gualtar

4710-057 Braga

Portugal

This article is available at http://intlpress.com/HHA/v8/n2/a1 